\title{
Interventions to Reduce Medication Dispensing, Administration, and Monitoring Errors in Pediatric Professional Healthcare Settings: A Systematic Review
}

\section{OPEN ACCESS}

Edited by:

Steven Hirschfeld,

Uniformed Services University of the Health Sciences, United States

Reviewed by:

Sara Garfield,

Imperial College London,

United Kingdom

Casper Bollen

University Medical Center

Utrecht, Netherlands

Allison Mari Dering-Anderson,

University of Nebraska Medical

Center, United States

*Correspondence:

Joachim A. Koeck

jkoeck@ukaachen.de

Specialty section:

This article was submitted to

Children and Health,

a section of the journal

Frontiers in Pediatrics

Received: 24 November 2020

Accepted: 12 April 2021

Published: 26 May 2021

Citation:

Koeck JA, Young NJ, Kontny U, Orlikowsky T, Bassler D and Eisert A

(2021) Interventions to Reduce

Medication Dispensing,

Administration, and Monitoring Errors in Pediatric Professional Healthcare

Settings: A Systematic Review.

Front. Pediatr. 9:633064.

doi: 10.3389/fped.2021.633064

\author{
Joachim A. Koeck ${ }^{1 *}$, Nicola J. Young ${ }^{1}$, Udo Kontny ${ }^{2}$, Thorsten Orlikowsky ${ }^{3}$, Dirk Bassler ${ }^{4}$ \\ and Albrecht Eisert ${ }^{1,5}$ \\ ${ }^{1}$ Hospital Pharmacy, Rheinisch-Westfälische Technische Hochschule Aachen University Hospital, Aachen, Germany, \\ ${ }^{2}$ Section of Pediatric Hematology, Department of Pediatric and Adolescent Medicine, Rheinisch-Westfälische Technische \\ Hochschule Aachen University Hospital, Aachen, Germany, ${ }^{3}$ Section of Neonatology, Department of Pediatric and \\ Adolescent Medicine, Rheinisch-Westfälische Technische Hochschule Aachen University Hospital, Aachen, Germany, \\ ${ }^{4}$ Department of Neonatology, University Hospital Zurich, Zurich, Switzerland, ${ }^{5}$ Institute of Clinical Pharmacology, University \\ Hospital of Rheinisch-Westfälische Technische Hochschule Aachen, Aachen, Germany
}

Introduction: Pediatric patients cared for in professional healthcare settings are at high risk of medication errors. Interventions to improve patient safety often focus on prescribing; however, the subsequent stages in the medication use process (dispensing, drug administration, and monitoring) are also error-prone. This systematic review aims to identify and analyze interventions to reduce dispensing, drug administration, and monitoring errors in professional pediatric healthcare settings.

Methods: Four databases were searched for experimental studies with separate control and intervention groups, published in English between 2011 and 2019. Interventions were classified for the first time in pediatric medication safety according to the "hierarchy of controls" model, which predicts that interventions at higher levels are more likely to bring about change. Higher-level interventions aim to reduce risks through elimination, substitution, or engineering controls. Examples of these include the introduction of smart pumps instead of standard pumps (a substitution control) and the introduction of mandatory barcode scanning for drug administration (an engineering control). Administrative controls such as guidelines, warning signs, and educational approaches are lower on the hierarchy and therefore predicted by this model to be less likely to be successful.

Results: Twenty studies met the inclusion criteria, including 1 study of dispensing errors, 7 studies of drug administration errors, and 12 studies targeting multiple steps of the medication use process. A total of 44 interventions were identified. Eleven of these were considered higher-level controls (four substitution and seven engineering controls). The majority of interventions ( $n=33$ ) were considered "administrative controls" indicating a potential reliance on these measures. Studies that implemented higher-level controls were observed to be more likely to reduce errors, confirming that the hierarchy of controls model may be useful in this setting. Heterogeneous study methods, definitions, and outcome measures meant that a meta-analysis was not appropriate. 
Conclusions: When designing interventions to reduce pediatric dispensing, drug administration, and monitoring errors, the hierarchy of controls model should be considered, with a focus placed on the introduction of higher-level controls, which may be more likely to reduce errors than the administrative controls often seen in practice. Trial Registration Prospero Identifier: CRD42016047127.

Keywords: medication error, medication safety, child, pediatric, dispensing error, administration error, monitoring error, hierarchy of controls

\section{INTRODUCTION}

The ongoing need to address medication safety and reduce the risks to patients from their medications was recently reinforced by the World Health Organization Technical Report, "Medication Safety in High-Risk Situations"; the report highlighted that adverse events resulting from medication errors are now estimated to be the 14th leading cause of morbidity and mortality in the world (1). In pediatric patients, pharmacokinetic and pharmacodynamic parameters can be significantly different from those in adults (2). As pediatric specific formulations are often not available, adult formulations have to be manipulated off-label before use (e.g., through crushing a tablet and taking a portion from it). This results in an additional risk for miscalculations (2). As a result, children are at an estimated three times higher risk of potential adverse drug events than adults (3).

Medication errors are broadly defined as "any preventable event that may cause or lead to inappropriate medication use or patient harm while the medication is in the control of the healthcare professional, patient, or consumer" (4). These events may occur in every step of the medication use process (MUP). Aitken et al. describe the MUP as four stages, starting with prescribing, followed by preparation and/or dispensing of the medication, and then drug administration and finally monitoring for both therapeutic and adverse effects (5). The MUP is cyclical, and depending on the outcomes of the monitoring process, the decision may be made to either stop a medication or issue a further prescription for the same or a different medication. It has been suggested that the drug administration step of the MUP is the most prone to error (5). Despite this, the current literature focuses on interventions to prevent and/or reduce prescribing errors, with a lesser focus on the subsequent stages of the MUP. As the last overview of these interventions was published in 2014 (incorporating data up to November 22, 2011) (6), a follow-on is needed. This review seeks to identify interventions designed to reduce and/or prevent drug dispensing, administration, and monitoring errors and determine their effect.

\section{METHODS}

\section{Protocol and Registration}

The protocol was registered in PROSPERO (reg. no. CRD42016047127) and with the local ethics commission (EK 158/17). To promote a differentiated focus on the individual stages of the MUP, the results of the review were split post-hoc, to consider interventions targeting prescribing errors in another publication. The structure of this article was guided by the recommendations of Preferred Reporting Items for Systematic Reviews and Meta-Analyses (PRISMA) (7).

\section{Eligibility Criteria}

Studies of interventions to reduce drug dispensing, administration, and/or monitoring errors in professional pediatric healthcare settings were included. An intervention was defined as any action or set of actions implemented with the aim of reducing medication errors. The definition of a medication error from the "National Coordinating Council for Medication Error Reporting and Prevention" was adopted, to provide a broad classification and allow for the inclusion of as many studies as possible (4). Definitions of dispensing errors, drug administration errors, and monitoring errors adopted for this review are provided in Table 1 along with their sources (8-10). A professional healthcare setting was defined as all inpatient and outpatient facilities where a healthcare professional is involved in the MUP. Medication errors occurring when the medication is in the care of the patient and/or their family are outside the scope of this systematic review.

Five study types were included. Definitions from the "Cochrane Effective Practice and Organization of Care Review Group" were adopted for "randomized controlled trial," "controlled clinical trial," "controlled before-after study," and "interrupted time-series study." The fifth study type included "uncontrolled before-after study," defined as a study involving a comparison of two patient groups, with and without the investigated intervention.

Outcome parameters defined for the analysis were as follows: intervention types and their impact on reducing dispensing, drug administration, and/or monitoring errors according to each article's assessment.

\section{Information Sources}

The search strategy was adapted from the previous systematic review by Rinke et al. (6) (see Supplementary Material 1).

\section{Search}

The analysis included the time span from November 22, 2011, to December 31, 2019, in order to provide a followon from the previous systematic review (6). Previously piloted terms were used to search CENTRAL, CINAHL, EMBASE, and MEDLINE. Reference searching in the bibliographies of each included article and selected reviews $(6,11-51)$ complemented the aforementioned search. 


\section{Study Selection}

One reviewer $(\mathrm{JK})$ assessed the resulting titles and abstracts using a piloted form adapted from the form initially used by Rinke et al. (6). Abstracts were evaluated according to 16 exclusion criteria (see Supplementary Table 1). If none of the criteria were met, the abstract was included in the screening of full texts against these criteria. A second reviewer (AE) independently examined a random $10 \%$ of the first reviewer's search results of each database using the same form. Interrater agreement was calculated via Cohen $\kappa(52)$.

\section{Data Collection Process}

Data extraction was performed using a piloted form, first by one (JK) and then by a second reviewer (AE). Results were discussed and amended if necessary. The results of the literature research were analyzed using Excel 2016 (Redmond, WA, USA).

\section{Data Items}

Data collection included the interventions that were tested and their reported effect, the study type, number and characteristics of included patients, and the type of healthcare professionals delivering the intervention(s).

\section{Risk of Bias in Individual Studies}

Bias risk was evaluated according to the COCHRANE tool for randomized controlled trials, controlled clinical trials, controlled before-after studies, and interrupted time-series studies (53, $54)$, whereas uncontrolled before-after studies were rated via ROBINS-I (55).

TABLE 1 | Definitions of error subtypes.

\begin{tabular}{|c|c|}
\hline Type of error & Definition \\
\hline $\begin{array}{l}\text { Medication } \\
\text { error (ME) }\end{array}$ & $\begin{array}{l}\text { "A medication error is any preventable event that may cause or } \\
\text { lead to inappropriate medication use or patient harm while the } \\
\text { medication is in the control of the healthcare professional, } \\
\text { patient, or consumer. Such events may be related to professional } \\
\text { practice, healthcare products, procedures, and systems, } \\
\text { including prescribing, order communication, product labeling, } \\
\text { packaging, and nomenclature, compounding, dispensing, } \\
\text { distribution, administration, education, monitoring, and use" (4). }\end{array}$ \\
\hline $\begin{array}{l}\text { Dispensing } \\
\text { error (DE) }\end{array}$ & $\begin{array}{l}\text { "Any unintended deviation from an interpretable written } \\
\text { prescription or medication order. Both content and labeling } \\
\text { errors are included. Any unintended deviation from professional } \\
\text { or regulatory references, or guidelines affecting dispensing } \\
\text { procedures, is also considered a dispensing error" (8). }\end{array}$ \\
\hline $\begin{array}{l}\text { Administration } \\
\text { error (AE) }\end{array}$ & $\begin{array}{l}\text { "Administration of a dose of medication that deviates from the } \\
\text { prescription, as written on the patient medication chart, or from } \\
\text { standard hospital policy and procedures. This includes errors in } \\
\text { the preparation and administration of intravenous medicines on } \\
\text { the ward" (9). }\end{array}$ \\
\hline $\begin{array}{l}\text { Monitoring } \\
\text { error (MO) }\end{array}$ & $\begin{array}{l}\text { "When a prescribed medicine is not monitored in the way that } \\
\text { would be considered acceptable in routine general practice. It } \\
\text { includes the absence of tests being carried out at the frequency } \\
\text { listed in the criteria, with tolerance of }+50 \% \text { " (10). This includes } \\
\text { monitoring after initiation and continuation of therapy. }\end{array}$ \\
\hline
\end{tabular}

\section{Summary Measures}

The impact of interventions was assessed through the calculation of the error rate (based on each author's definition of a medication error) for the control and intervention groups. Based on this, "absolute risk reduction" was established for each single intervention or bundle of interventions. When insufficient data were available to calculate separate error ratios for two study groups, this was not undertaken, and the ratio of error before and after intervention was calculated, based on the ratios given by individual study authors (Table 2 ).

\section{Synthesis of Results}

Authors classified studies as either a "single-intervention study" or a "bundle-intervention study," according to whether or not they implemented single or multiple interventions. The step(s) in the MUP targeted by the interventions were identified, and studies were also classified according to the errors they seek to reduce, either dispensing, drug administration, or monitoring errors individually or when they target multiple steps in the MUP as investigating "combined medication errors." For combined medication error studies, the impact of interventions on MUP steps other than prescribing was extracted for this analysis.

The individual interventions were classified according to a hierarchical approach to risk control (76). It has been suggested that this approach can aid in the identification of appropriate interventions to reduce known risks occurring during riskprone processes, and it has been hypothesized that this approach could be successfully adopted in the healthcare setting (77). The highest (most likely to be successful) level of control involves eliminating the risk entirely; the next level suggests making a substitution, so that a risk is reduced when it is not possible to eliminate it (e.g., the substitution of manually operated infusion pumps with smart pumps containing a drug database). Engineering controls are next on the hierarchy and involve attempts to isolate a risk or to isolate a patient from a risk. For example, barcoded drug administration is an engineering control, which aims to reduce the risks of patients receiving the wrong medication via mandatory scanning a patient wristband and/or the medication to be given; if either is false, this is highlighted to the healthcare professional administering the medication, and they are prevented from proceeding to document the administration. This step is followed by administrative controls, which often include informative signage and education; personal protective equipment completes the hierarchical approach, as the lowest level of control. Figure 1 displays the "hierarchy of controls" with medication safety-related examples for each level. For analysis, elimination, substitution, and engineering controls were combined and considered as higher-level controls, to be compared with the lower levels of control (administrative controls and personal protective equipment). This division into higher- and lower-level controls was performed in analogy to the methods presented by Card et al. (78).

The classification of interventions was performed independently by two authors (JK, NY). Interrater agreement was calculated via weighted Cohen $\kappa(52)$. Discrepancies were discussed and resolved by a third author (AE) when necessary. 
TABLE 2 | Summary of study characteristics.

Studies addressing one step in the medication use process

\begin{tabular}{|c|c|c|c|c|c|c|c|c|c|}
\hline $\begin{array}{l}\text { First author } \\
\text { (country, year) }\end{array}$ & Error type(s) & $\begin{array}{l}\text { Study design, } \\
\text { study centers }\end{array}$ & Setting & Methods & Intervention & $\begin{array}{l}\text { Single/bundle } \\
\text { intervention }\end{array}$ & Results & Error rate $\mathrm{CG} / \mathrm{IG}$ & $\begin{array}{l}\text { Absolute risk } \\
\text { reduction } \\
\text { (reported level of } \\
\text { significance) }\end{array}$ \\
\hline $\begin{array}{l}\text { Campino et al. } \\
\text { (Spain, 2016) (56) }\end{array}$ & $\begin{array}{l}\text { Drug } \\
\text { administration } \\
\text { errors }\end{array}$ & UBA, multicenter & $\begin{array}{l}10 \mathrm{NICU} \text { and } \\
\text { one hospital } \\
\text { pharmacy }\end{array}$ & $\begin{array}{l}\text { Assessment of randomly } \\
\text { collected intravenous dilutions } \\
\text { of vancomycin, gentamicin, } \\
\text { phenobarbital, and caffeine } \\
\text { citrate to investigate } \\
\text { calculation and accuracy } \\
\text { errors during preparation; } \\
\text { most dilutions contained } \\
\text { vancomycin or gentamicin } \\
\text { (75\% and } 71 \% \text {, respectively) }\end{array}$ & $\begin{array}{l}\text { (1) Standardized preparation } \\
\text { protocol with no need } \\
\text { for calculation; } \\
\text { (2) Educational program, } \\
\text { developed by pharmacists, } \\
\text { nurses, and physicians } \\
\text { including preinterventional } \\
\text { results, basic rules for } \\
\text { medication preparation, } \\
\text { weak points of medication } \\
\text { preparation, and new } \\
\text { preparation protocols; } \\
\text { education was repeated } \\
\text { several times }\end{array}$ & Bundle & $\begin{array}{l}\text { Calculation errors: } \\
\text { CG: 6, IG:0; all } \\
\text { NICUs/hospital } \\
\text { pharmacy benefited } \\
\text { from the intervention } \\
\text { (but to different } \\
\text { degrees); total: } 266 \\
\text { accuracy errors } \\
\text { (underdosing/overdosing)/ } \\
\text { preparations, IG: } 67 / 332\end{array}$ & $\begin{array}{l}52.78 \% / 21.99 \% \\
\\
/ 504\end{array}$ & $\begin{array}{c}30.79 \% \\
\text { (significantly } \\
\text { positive results) }\end{array}$ \\
\hline $\begin{array}{l}\text { Chedoe et al. } \\
\text { (Netherlands, 2012) } \\
\text { (57) }\end{array}$ & $\begin{array}{c}\text { Drug } \\
\text { administration } \\
\text { errors }\end{array}$ & $\begin{array}{l}\text { UBA, single } \\
\text { center }\end{array}$ & $\mathrm{NICU}$ & $\begin{array}{l}\text { Direct observation of } \\
\text { ward-based preparation and } \\
\text { application of drugs by trained } \\
\text { pharmacy students for } 10 \\
\text { consecutive days ( } 24 \mathrm{~h} / \text { day; } \\
\text { preinterventional and } \\
\text { post-interventional, } \\
\text { respectively); development of } \\
\text { a standardized data collection } \\
\text { form in pilot phase; each } \\
\text { medication dose observation } \\
\text { could contain more than one } \\
\text { error }\end{array}$ & $\begin{array}{l}\text { Educational program } \\
\text { consisting of an } 1-\mathrm{h} \\
\text { teaching session and } \\
\text { 30-min individual practical } \\
\text { training for preparation and } \\
\text { administration of commonly } \\
\text { used medications; } \\
\text { education was repeated } \\
\text { three times }\end{array}$ & Single & $\begin{array}{c}\text { CG: } 151 \text { doses with } \geq 1 \\
\text { error/311 observed } \\
\text { doses; IG: 87/284; } \\
\text { clinical relevance was } \\
\text { assessed by three } \\
\text { experts (pharmacist, } \\
\text { neonatologist, } \\
\text { neonatological nurse); } \\
\text { interventional effects } \\
\text { addressed } \\
\text { administration errors } \\
\text { mainly }\end{array}$ & $48.55 \% / 30.63 \%$ & $\begin{array}{c}17.92 \% \\
\text { (significantly } \\
\text { positive results) }\end{array}$ \\
\hline $\begin{array}{l}\text { Chua et al. } \\
\text { (Malaysia, 2017) } \\
\text { (58) }\end{array}$ & $\begin{array}{c}\text { Drug } \\
\text { administration } \\
\text { errors }\end{array}$ & $\begin{array}{l}\text { UBA, single } \\
\text { center }\end{array}$ & $\begin{array}{l}\text { Pediatric } \\
\text { ward }\end{array}$ & $\begin{array}{l}\text { Direct observation of drug } \\
\text { administration by pharmacists } \\
\text { on two pediatric wards for } 40 \\
\text { days preintervention and } \\
\text { post-intervention, respectively }\end{array}$ & $\begin{array}{l}\text { Pharmacist-led presentation } \\
\text { of preintervention drug } \\
\text { administration errors to } \\
\text { pediatric physicians and } \\
\text { nurses, followed by } \\
\text { discussion (repeated six } \\
\text { times) }\end{array}$ & Single & $\begin{array}{l}\text { Pre: } 1,284 \text { doses } \\
\text { observed for } 217 \\
\text { patients (5.9 doses per } \\
\text { patient) with } 569 \text { doses } \\
\text { containing at least one } \\
\text { error ( } 852 \text { errors total); } \\
\text { post: } 1,401 \text { doses for } \\
208 \text { patients ( } 6.7 \text { doses } \\
\text { per patient) with } 400 \\
\text { doses containing at } \\
\text { least one error ( } 496 \\
\text { errors total) }\end{array}$ & $44.31 \% / 28.55 \%$ & $\begin{array}{c}15.76 \% \\
\text { (significantly } \\
\text { positive results) }\end{array}$ \\
\hline
\end{tabular}


Studies addressing one step in the medication use process

\begin{tabular}{|c|c|c|c|c|c|c|c|c|c|}
\hline $\begin{array}{l}\text { First author } \\
\text { (country, year) }\end{array}$ & Error type(s) & $\begin{array}{l}\text { Study design, } \\
\text { study centers }\end{array}$ & Setting & Methods & Intervention & $\begin{array}{l}\text { Single/bundle } \\
\text { intervention }\end{array}$ & Results & Error rate $\mathrm{CG} / \mathrm{IG}$ & $\begin{array}{l}\text { Absolute risk } \\
\text { reduction } \\
\text { (reported level of } \\
\text { significance) }\end{array}$ \\
\hline $\begin{array}{l}\text { Davis et al. (USA, } \\
\text { 2017) (59) }\end{array}$ & $\begin{array}{l}\text { Dispensing } \\
\text { errors }\end{array}$ & $\begin{array}{l}\text { UBA, single } \\
\text { center }\end{array}$ & $\begin{array}{l}\text { Hospital } \\
\text { pharmacy }\end{array}$ & $\begin{array}{l}\text { Implementation of an } \\
\text { electronic work flow } \\
\text { management system. The } \\
\text { system interfaced with the } \\
\text { computerized physician order } \\
\text { entry }\end{array}$ & $\begin{array}{l}\text { Electronic workflow } \\
\text { management system }\end{array}$ & Single & $\begin{array}{c}\text { Pre: } 9.8 \text { errors per } \\
\text { 10,000 doses } \\
\text { dispensed; post: } 8.2 \\
\text { errors per } 10,000 \text { doses }\end{array}$ & 0.098\%/0.082\% & $\begin{array}{c}0.016 \% \\
\text { (significantly } \\
\text { positive results) }\end{array}$ \\
\hline $\begin{array}{l}\text { Marconi et al. (USA, } \\
\text { 2012) (60) }\end{array}$ & $\begin{array}{l}\text { Drug } \\
\text { administration } \\
\text { errors }\end{array}$ & $\begin{array}{l}\text { UBA, single } \\
\text { center }\end{array}$ & EP & $\begin{array}{l}\text { Assessment of missed or } \\
\text { delayed ( }>1 \mathrm{~h} \text { later than } \\
\text { scheduled time) medication } \\
\text { administrations in the } \\
\text { emergency department; } \\
\text { separation of medications into } \\
\text { "urgent" (medication for } \\
\text { patients' chief complaint or } \\
\text { acute diagnosis) and } \\
\text { non-urgent medications }\end{array}$ & $\begin{array}{c}\text { Emergency department } \\
\text { pharmacist }\end{array}$ & Single & $\begin{array}{c}\text { CG: } 29 \text { urgent and } 169 \\
\text { non-urgent } \\
\text { medications/723 } \\
\text { medications; IG: } 21 \\
\text { urgent and } 64 \\
\text { non-urgent } \\
\text { medications } / 851 \\
\text { medications }\end{array}$ & $\begin{array}{c}\text { Missed or delayed } \\
\text { medications total: } \\
27.39 \% / 9.99 \%\end{array}$ & $\begin{array}{c}\text { Missed or delayed } \\
\text { medications total: } \\
17.40 \% \\
\text { (significantly } \\
\text { positive results) }\end{array}$ \\
\hline $\begin{array}{l}\text { Niemann et al. } \\
\text { (Germany, 2015) } \\
\text { (61) }\end{array}$ & $\begin{array}{l}\text { Drug } \\
\text { administration } \\
\text { errors }\end{array}$ & $\begin{array}{l}\text { UBA, single } \\
\text { center }\end{array}$ & $\begin{array}{l}\text { Pediatric } \\
\text { ward }\end{array}$ & $\begin{array}{l}\text { Direct observation by four } \\
\text { trained pharmacists using a } \\
\text { predefined } 22 \text {-item list of } \\
\text { drug-handling processes; } \\
\text { monitoring in the morning } \\
\text { (7:30-10:00 a.m.) for } 20 \\
\text { working days }\end{array}$ & $\begin{array}{l}\text { Three-step educational } \\
\text { intervention: three-page } \\
\text { handout (addressed } \\
\text { knowledge deficits and } \\
\text { memory-based lapses), } \\
60 \text {-min pharmacist-led } \\
\text { education (background } \\
\text { information and } \\
\text { drug-handling guidelines), } \\
\text { and 56-page } \\
\text { comprehensive reference } \\
\text { book (detailed information } \\
\text { about drug-handling) }\end{array}$ & Single & $\begin{array}{l}\text { Patients: CG: } 38 / 43 \\
\text { patients suffered } \geq 1 \mathrm{ME} \\
\text { within the observed } \\
\text { processes; IG: } 25 / 51 ; \\
\text { processes: } \mathrm{CG} \text { : } \\
\text { 370/581 observed } \\
\text { processes contained } \geq 1 \\
\text { error; IG: } 100 / 441\end{array}$ & $\begin{array}{c}\text { Patients: } \\
\text { 88.37\%/49.02\%; } \\
\text { processes: } \\
63.68 \% / 22.68 \% \\
\end{array}$ & $\begin{array}{c}\text { Patients: } 39.35 \% \text {; } \\
\text { processes: } 41.00 \% \\
\text { (significantly } \\
\text { positive results) }\end{array}$ \\
\hline $\begin{array}{l}\text { Niemann et al. } \\
\text { (Germany, 2014) } \\
\text { (62) }\end{array}$ & $\begin{array}{l}\text { Drug } \\
\text { administration } \\
\text { errors }\end{array}$ & $\begin{array}{l}\text { UBA, single } \\
\text { center }\end{array}$ & PICU & $\begin{array}{l}\text { Direct observation by five } \\
\text { trained pharmacists using a } \\
\text { pre-defined } 24 \text {-item-list of } \\
\text { drug-handling processes; } \\
\text { monitoring between } \\
\text { 7:00-9:00 a.m. and } \\
\text { 11:00 a.m. to 1:00 p.m. for } 26 \\
\text { working days }\end{array}$ & $\begin{array}{l}\text { Three-step educational } \\
\text { intervention: three-page } \\
\text { handout, } 60 \text {-min } \\
\text { pharmacist-led education, } \\
\text { and } 76 \text {-page } \\
\text { comprehensive reference } \\
\text { book }\end{array}$ & Single & $\begin{array}{c}\text { Patients: CG: } 36 / 38 \\
\text { patients suffered } \geq 1 \mathrm{ME} \\
\text { within the observed } \\
\text { processes; IG: } 42 / 47 ; \\
\text { processes: CG: } \\
\text { 384/668 observed } \\
\text { processes contained } \geq 1 \\
\text { error; IG: } 445 / 883\end{array}$ & $\begin{array}{c}\text { Patients: } \\
\text { 94.74\%/89.36\%; } \\
\text { processes: } \\
57.49 \% / 50.40 \% \\
\end{array}$ & $\begin{array}{l}\text { Patients: 5.38\%; } \\
\text { processes: } 7.09 \% \\
\text { (mixed results) }\end{array}$ \\
\hline
\end{tabular}


TABLE 2 | Continued

Studies addressing one step in the medication use process

\begin{tabular}{|c|c|c|c|c|c|c|c|c|c|}
\hline $\begin{array}{l}\text { First author } \\
\text { (country, year) }\end{array}$ & Error type(s) & $\begin{array}{l}\text { Study design, } \\
\text { study centers }\end{array}$ & Setting & Methods & Intervention & $\begin{array}{l}\text { Single/bundle } \\
\text { intervention }\end{array}$ & Results & Error rate $\mathrm{CG} / \mathrm{IG}$ & $\begin{array}{l}\text { Absolute risk } \\
\text { reduction } \\
\text { (reported level of } \\
\text { significance) }\end{array}$ \\
\hline $\begin{array}{l}\text { Ozkan et al. } \\
\text { (Turkey, 2013) (63) }\end{array}$ & $\begin{array}{l}\text { Drug } \\
\text { administration } \\
\text { errors }\end{array}$ & $\begin{array}{l}\text { UBA, single } \\
\text { center }\end{array}$ & $\begin{array}{l}\text { Pediatric } \\
\text { ward }\end{array}$ & $\begin{array}{c}\text { Direct Observation of } \\
\text { medication administration } \\
\text { (observation period between } \\
\text { 10:00 a.m. - 6:00 p.m. and } \\
\text { 10:00 p.m. - 6:00 a.m.); } \\
\text { assessment of a deviation } \\
\text { between the physician's order } \\
\text { and the administered } \\
\text { medication }\end{array}$ & $\begin{array}{l}\text { (1) Written alerts displayed } \\
\text { on the door of the } \\
\text { preparation room } \\
\text { (2) Signaling arm bands for } \\
\text { the medication preparing } \\
\text { and administration nurses } \\
\text { (3) Earlier medication } \\
\text { delivery by pharmacy } \\
\text { (4) Preparation and } \\
\text { administration guidelines } \\
\text { (5) Increase } \\
\text { of nurse/patient-ratio }\end{array}$ & Bundle & $\begin{array}{l}\text { CG: } 475 \text { errors/1,686 } \\
\text { observed medication } \\
\text { doses; IG: } 313 / 1,460 ; \\
\text { workload determined as } \\
\text { leading cause for } \\
\text { administration errors (no } \\
\text { significant difference } \\
\text { between CG and IG) }\end{array}$ & $28.17 \% / 21.44 \%$ & $\begin{array}{l}6.73 \% \text { (significantly } \\
\text { positive results) }\end{array}$ \\
\hline
\end{tabular}

Studies addressing multiple steps in the medication use process (combined medication errors)

\begin{tabular}{|c|c|c|c|c|c|c|c|c|c|}
\hline $\begin{array}{l}\text { Abuelsoud (Egypt, } \\
\text { 2019) (64) }\end{array}$ & $\begin{array}{c}\text { Combined } \\
\text { medication } \\
\text { errors } \\
\text { (prescribing, } \\
\text { drug } \\
\text { administration, } \\
\text { monitoring } \\
\text { errors) }\end{array}$ & $\begin{array}{l}\text { UBA, single } \\
\text { center }\end{array}$ & $\begin{array}{l}\text { Pediatric } \\
\text { ward }\end{array}$ & $\begin{array}{l}\text { FOCUS-PDCA technique } \\
\text { (Plan-Do-Study-Act cycle); } \\
100 \text { medical files were } \\
\text { randomly selected on a } \\
\text { pediatric medical ward per } \\
\text { month over a period of } 9 \\
\text { months; it was aimed at } \\
\text { reducing drug-related } \\
\text { problems in each of the three } \\
\text { selected steps of the } \\
\text { medication use process } \\
\text { prescribing, administration, } \\
\text { and monitoring steps to } \\
\leq 15 \% \text { within } 9 \text { months }\end{array}$ & $\begin{array}{l}\text { (1) Conducting an } \\
\text { educational program to } \\
\text { pediatric staff (physicians } \\
\text { and nurses) } \\
\text { (2) Implementation of a } \\
\text { clinical pharmacist into the } \\
\text { medical team } \\
\text { (3) Establishment of drug } \\
\text { information center } \\
\text { (4) Establishment of IV } \\
\text { admixture unit } \\
\text { (5) Using auxiliary labels }\end{array}$ & Bundle & $\begin{array}{l}900 \text { medical files } \\
\text { reviewed (100 files per } \\
\text { month); prescribing } \\
\text { errors: CG (1st month): } \\
47 \text { errors/ } 100 \text { files, IG } \\
\text { (9th month): } 10 \text { errors/ } \\
100 \text { files; drug } \\
\text { administration errors: } \\
\text { CG (1st month): } 60 \\
\text { errors/ } 100 \text { files, IG (9th } \\
\text { month): } 10 \text { errors/ } 100 \\
\text { files; monitoring errors: } \\
\text { CG (1st month): } 56 \\
\text { errors/ } 100 \text { files, IG (9th } \\
\text { month): } 15 \text { errors/ } 100 \\
\text { files }\end{array}$ & $\begin{array}{c}\text { Administration } \\
\text { errors: } 60 \% / 10 \% \text {; } \\
\text { monitoring errors: } \\
56 \% / 15 \%\end{array}$ & $\begin{array}{l}\text { Administration } \\
\text { errors: } 50 \% \text {; } \\
\text { monitoring errors: } \\
41 \% \text { (significantly } \\
\text { positive results) }\end{array}$ \\
\hline
\end{tabular}


TABLE 2 | Continued

Studies addressing multiple steps in the medication use process (combined medication errors)

\begin{tabular}{|c|c|c|c|c|c|c|c|c|c|}
\hline $\begin{array}{l}\text { First author } \\
\text { (country, year) }\end{array}$ & Error type(s) & $\begin{array}{l}\text { Study design, } \\
\text { study centers }\end{array}$ & Setting & Methods & Intervention & $\begin{array}{l}\text { Single/bundle } \\
\text { intervention }\end{array}$ & Results & Error rate $\mathrm{CG} / \mathrm{IG}$ & $\begin{array}{l}\text { Absolute risk } \\
\text { reduction } \\
\text { (reported level of } \\
\text { significance) }\end{array}$ \\
\hline $\begin{array}{l}\text { Benkelfat et al. } \\
\text { (Canada, 2013) (65) }\end{array}$ & $\begin{array}{l}\text { Combined } \\
\text { medication } \\
\text { errors } \\
\text { (prescribing } \\
\text { errors, drug } \\
\text { administration } \\
\text { errors) }\end{array}$ & $\begin{array}{l}\text { CCT, single } \\
\text { center }\end{array}$ & EP & $\begin{array}{l}\text { Retrospective ME analysis of } \\
\text { medical records (drug choice, } \\
\text { dosage deviation }>10 \% \text { of } \\
\text { recommended dosing, } \\
\text { frequency, and route of } \\
\text { administration) for children } \\
<18 \text { years, who were treated } \\
\text { for anaphylaxis in the } \\
\text { emergency department }\end{array}$ & $\begin{array}{l}\text { Standard order form for } \\
\text { medications used in } \\
\text { anaphylaxis }\end{array}$ & Single & $\begin{array}{l}\text { CG: } 18 \text { medical charts } \\
\text { with } \geq 1 \text { error/30 } \\
\text { medical charts; IG: } \\
\text { 16/29; dosing errors } \\
\text { were significantly } \\
\text { reduced, but not errors } \\
\text { in total }\end{array}$ & $60.00 \% / 55.17 \%$ & $\begin{array}{l}\text { 4,83\% (significantly } \\
\text { positive results) }\end{array}$ \\
\hline $\begin{array}{l}\text { Ernst et al. (USA, } \\
\text { 2017) (66) }\end{array}$ & $\begin{array}{l}\text { Combined } \\
\text { medication } \\
\text { errors } \\
\text { (prescribing } \\
\text { errors, drug } \\
\text { administration } \\
\text { errors) }\end{array}$ & $\begin{array}{l}\text { UBA, single } \\
\text { center }\end{array}$ & $\mathrm{NICU}$ & $\begin{array}{l}\text { A retrospective EMR chart } \\
\text { review of children with a birth } \\
\text { weight }<2 \mathrm{~kg} \text { and a } \\
\text { hospitalization of } \geq 58 \text { days } \\
\text { were included in the time } \\
\text { range of 2009-2013. The } \\
\text { 2-month immunization status } \\
\text { was investigated for the seven } \\
\text { vaccines recommended }\end{array}$ & $\begin{array}{l}\text { An electronic immunization } \\
\text { alert was introduced into the } \\
\text { EMR. It was shown from } \\
\text { days } 56 \text { to } 67 \text { on the } \\
\text { beginning of the day to the } \\
\text { physicians and nurses } \\
\text { separately }\end{array}$ & Single & $\begin{array}{l}\text { CG: } 35 \text { infants partially } \\
\text { immunized or } \\
\text { unimmunized/121 } \\
\text { infants; IG: } 6 \text { infants } \\
\text { partially immunized or } \\
\text { unimmunized/140 } \\
\text { infants }\end{array}$ & 29\%/6\% & $\begin{array}{l}\text { 23\% (significantly } \\
\text { positive results) }\end{array}$ \\
\hline $\begin{array}{l}\text { Fawaz et al. (Egypt, } \\
\text { 2017) (67) }\end{array}$ & $\begin{array}{l}\text { Combined } \\
\text { medication } \\
\text { errors } \\
\text { (prescribing, } \\
\text { transcribing, and } \\
\text { drug } \\
\text { administration } \\
\text { errors) }\end{array}$ & $\begin{array}{l}\text { UBA, single } \\
\text { center }\end{array}$ & $\begin{array}{l}\text { Operating } \\
\text { room }\end{array}$ & $\begin{array}{l}\text { Pharmacist observed drug } \\
\text { handling in the operating } \\
\text { room and reviewed } \\
\text { prescribed medications }\end{array}$ & $\begin{array}{l}\text { Pharmacist-led educational } \\
\text { program consisting of } \\
\text { detection, reporting, and } \\
\text { prevention of medication } \\
\text { errors }\end{array}$ & Single & $\begin{array}{c}\text { Pre: } 312 \text { medication } \\
\text { errors were detected in } \\
110 \text { patients with } 936 \\
\text { medication orders ( } 6.2 \\
\text { medication orders per } \\
\text { patient); post: } 224 \\
\text { medication errors in } 112 \\
\text { patients with } 693 \\
\text { medication orders ( } 8.5 \\
\text { medication orders per } \\
\text { patient) }\end{array}$ & $33.33 \% / 32.32 \%$ & $\begin{array}{c}1.01 \% \\
\text { (non-significant } \\
\text { positive results) }\end{array}$ \\
\hline $\begin{array}{l}\text { Foster et al. (USA, } \\
\text { 2013) (68) }\end{array}$ & $\begin{array}{l}\text { Combined } \\
\text { medication } \\
\text { errors } \\
\text { (prescribing } \\
\text { errors, drug } \\
\text { administration } \\
\text { errors) }\end{array}$ & $\begin{array}{l}\text { ITS, single } \\
\text { center }\end{array}$ & EP & $\begin{array}{l}\text { Ward pharmacist reviewed } \\
\text { medication orders in the } \\
\text { emergency department on } \\
\text { weekdays from } 3 \text { to } 11 \text { pm; } \\
\text { assessment of ME rates in } \\
\text { three 3-month intervals }\end{array}$ & $\begin{array}{l}\text { 3-h educational program for } \\
\text { emergency department } \\
\text { residents, led by an } \\
\text { attending physician and the } \\
\text { ward pharmacist }\end{array}$ & Single & $\begin{array}{l}2 \text { of } 10 \text { investigated } \\
\text { drug-related problems } \\
\text { showed significant } \\
\text { improvements (dose } \\
\text { adjustment and order } \\
\text { clarification) }\end{array}$ & $\mathrm{N} / \mathrm{A}$ & N/A (mixed results) \\
\hline
\end{tabular}


Studies addressing multiple steps in the medication use process (combined medication errors)

\begin{tabular}{|c|c|c|c|c|c|c|c|c|c|}
\hline $\begin{array}{l}\text { First author } \\
\text { (country, year) }\end{array}$ & Error type(s) & $\begin{array}{l}\text { Study design, } \\
\text { study centers }\end{array}$ & Setting & Methods & Intervention & $\begin{array}{c}\text { Single/bundle } \\
\text { intervention }\end{array}$ & Results & Error rate $\mathrm{CG} / \mathrm{IG}$ & $\begin{array}{l}\text { Absolute risk } \\
\text { reduction } \\
\text { (reported level of } \\
\text { significance) }\end{array}$ \\
\hline $\begin{array}{l}\text { Keiffer et al. (USA, } \\
\text { 2015) (69) }\end{array}$ & $\begin{array}{c}\text { Combined } \\
\text { medication } \\
\text { errors } \\
\text { (prescribing } \\
\text { errors, drug } \\
\text { administration } \\
\text { errors) }\end{array}$ & $\begin{array}{l}\text { UBA, single } \\
\text { center }\end{array}$ & $\begin{array}{c}\text { Pediatric } \\
\text { cardiothoracic } \\
\text { intensive care } \\
\text { unit }\end{array}$ & $\begin{array}{l}\text { Analysis of MEs that resulted } \\
\text { in patient harm (NCC MERP } \\
\text { type D-I) through assessment } \\
\text { of voluntary reports by } \\
\text { pharmacists, trigger tools, } \\
\text { and hospital-wide voluntary } \\
\text { incident reports by } \\
\text { hospital-wide and unit-based } \\
\text { quality leaders }\end{array}$ & $\begin{array}{l}\text { (1) Quality process } \\
\text { education ("the 5-rights") } \\
\text { (2) Nursing independent } \\
\text { double check using a } \\
\text { standardized checklist; } \\
\text { (3) Hands-free } \\
\text { communication with } \\
\text { wearable } \\
\text { voice-controlled devices } \\
\text { (4) ME huddles } \\
\text { (5) A "distraction-free zone" } \\
\text { consisting of a physical mat } \\
\text { in front of the PYXIS and } \\
\text { signs placed on the } \\
\text { computers in the unit } \\
\text { (6) Bedside } \\
\text { medication barcoding }\end{array}$ & Bundle & $\begin{array}{c}33 \text { pADEs that resulted } \\
\text { in patient harm in 2010; } \\
3 \text { pADEs in 2011; } 6 \\
\text { pADEs in 2012; and } 4 \\
\text { pADEs in 2013; } \\
\text { harm-causing pADEs } \\
\text { were reduced from } 0.43 \\
\text { to } 0.05 \text { per } 1,000 \\
\text { administered medication } \\
\text { doses }\end{array}$ & $\begin{array}{l}\text { N/A [88\% error } \\
\text { reduction from CG } \\
\text { (2010) to IG } \\
\text { (2013)] }\end{array}$ & $\begin{array}{l}\text { N/A (significantly } \\
\text { positive results) }\end{array}$ \\
\hline $\begin{array}{l}\text { Maaskant et al. } \\
\text { (Netherlands, 2018) } \\
\text { (70) }\end{array}$ & $\begin{array}{l}\text { Combined } \\
\text { medication } \\
\text { errors } \\
\text { (prescribing, } \\
\text { drug } \\
\text { administration, } \\
\text { monitoring } \\
\text { errors) }\end{array}$ & $\begin{array}{l}\text { ITS, single } \\
\text { center }\end{array}$ & $\mathrm{PICU}$ & $\begin{array}{l}\text { Clinical records and the } \\
\text { incident reporting system of a } \\
\text { PICU were reviewed for } \\
\text { medication errors. When an } \\
\text { error was suspected, a } \\
\text { pediatric intensivist, and a } \\
\text { clinical pharmacist reviewed } \\
\text { the case. Six timepoints } \\
\text { preintervention and } \\
\text { post-intervention, respectively }\end{array}$ & $\begin{array}{l}\text { Pharmacist-led structured } \\
\text { medication audit and } \\
\text { feedback to pediatric } \\
\text { intensivists on a PICU. A } \\
\text { clinical pharmacist was } \\
\text { present on ward } 3 \mathrm{~h} 5 \text { days } \\
\text { per week. PICU-patients } \\
\text { with "(a) reduced renal } \\
\text { and/or hepatic clearance, } \\
\text { (b) oncological diagnoses, } \\
\text { (c) high-alert medication } \\
\text { prescriptions, (d) receiving } \\
\text { more than } 5 \text { medications, } \\
\text { and (e) medication } \\
\text { prescriptions with which the } \\
\text { PICU professionals felt } \\
\text { unfamiliar" were included. }\end{array}$ & Single & $\begin{array}{l}\text { Pre: within } 1 \text { year, } 254 \\
\text { patients were admitted } \\
\text { to the PICU with } 153 \\
\text { medication errors ( } 2.27 \\
\text { medication errors per } \\
100 \text { prescriptions; } 23 \\
\text { harmful medication } \\
\text { errors); post: within } 1 \\
\text { year, } 230 \text { patients were } \\
\text { admitted with } 90 \\
\text { medication errors (1.74 } \\
\text { medication errors per } \\
100 \text { prescriptions; } 6 \\
\text { harmful medication } \\
\text { errors), } 75 \text { of these } 230 \\
\text { patients were audited by } \\
\text { the pharmacist: the } \\
\text { prevalence of } \\
\text { medication errors was } \\
\text { found significantly lower } \\
\text { in patients with } \\
\text { medication audit }\end{array}$ & $2.27 \% / 1.74 \%$ & $\begin{array}{l}0.53 \% \text { (significantly } \\
\text { positive results) }\end{array}$ \\
\hline
\end{tabular}


Studies addressing multiple steps in the medication use process (combined medication errors)

\begin{tabular}{|c|c|c|c|c|c|c|c|c|c|}
\hline $\begin{array}{l}\text { First author } \\
\text { (country, year) }\end{array}$ & Error type(s) & $\begin{array}{l}\text { Study design, } \\
\text { study centers }\end{array}$ & Setting & Methods & Intervention & $\begin{array}{l}\text { Single/bundle } \\
\text { intervention }\end{array}$ & Results & Error rate $\mathrm{CG} / \mathrm{IG}$ & $\begin{array}{l}\text { Absolute risk } \\
\text { reduction } \\
\text { (reported level of } \\
\text { significance) }\end{array}$ \\
\hline $\begin{array}{l}\text { Martin et al. (USA, } \\
\text { 2017) (71) }\end{array}$ & $\begin{array}{l}\text { Combined } \\
\text { medication } \\
\text { errors } \\
\text { (prescribing, } \\
\text { drug } \\
\text { administration, } \\
\text { monitoring } \\
\text { errors) }\end{array}$ & $\begin{array}{l}\text { UBA, single } \\
\text { center }\end{array}$ & $\begin{array}{l}\text { Operating } \\
\text { room }\end{array}$ & $\begin{array}{l}\text { Anesthesiologists were } \\
\text { directly observed for } 2 \\
\text { months regarding the drug } \\
\text { handling and medication } \\
\text { errors in the operating room. } \\
\text { Using this data, a } \\
\text { failure-mode-and-effect } \\
\text { analysis was performed to } \\
\text { develop interventions, } \\
\text { followed by a reobservation }\end{array}$ & $\begin{array}{l}\text { (1) Medication tray } \\
\text { reorganization } \\
\text { (pharmacy-prepared trays, } \\
\text { reorganization with colors } \\
\text { and sequesters; due to } \\
\text { high-risk medications, and } \\
\text { according to the frequency } \\
\text { of usage) } \\
\text { (2) Medication cart top } \\
\text { template (standardized } \\
\text { organization of } \\
\text { common medications) } \\
\text { (3) Syringe labeling } \\
\text { (standard nomenclature } \\
\text { and color-coding) } \\
\text { (4) Infusion double check } \\
\text { (independent double check, } \\
\text { documented with preprinted } \\
\text { labeling tape on the infusion) } \\
\text { (5) Medication practice } \\
\text { guideline (developed and } \\
\text { posted in every operating } \\
\text { room: syringe labeling, } \\
\text { medication preparation) }\end{array}$ & Bundle & $\begin{array}{l}\text { Pre: } 368 \text { syringes for } 68 \\
\text { patients were audited } \\
\text { with } 101 \text { labeling errors } \\
\text { within } 2 \text { months; } 17 \\
\text { infusion pumps were } \\
\text { checked with 13-times } \\
\text { double-check error. No } \\
\text { standardized workspace } \\
\text { organization was found; } \\
\text { post: } 402 \text { syringes for } \\
61 \text { patients were } \\
\text { audited with } 16 \text { labeling } \\
\text { errors within } 2 \text { months. } \\
17 \text { infusion pumps were } \\
\text { checked with } 7 \text {-times } \\
\text { double-check error }\end{array}$ & $\begin{array}{c}\text { Labeling error: } \\
27.4 \% / 4.0 \% \text {; } \\
\text { infusion double } \\
\text { check: } \\
76.5 \% / 41.2 \%\end{array}$ & $\begin{array}{l}\text { Labeling error: } \\
\text { 23.5\%; infusion } \\
\text { double check: } \\
\begin{array}{c}35.3 \% \text { (mixed } \\
\text { results) }\end{array}\end{array}$ \\
\hline $\begin{array}{l}\text { McClead et al. } \\
\text { (USA, 2014) (72) }\end{array}$ & $\begin{array}{l}\text { Combined } \\
\text { medication } \\
\text { errors } \\
\text { (prescribing-, } \\
\text { drug } \\
\text { administration-, } \\
\text { dispensing } \\
\text { errors) }\end{array}$ & $\begin{array}{l}\text { UBA, single } \\
\text { center }\end{array}$ & $\begin{array}{l}\text { Entire hospital } \\
\text { (1) }\end{array}$ & $\begin{array}{l}\text { Within a pediatric hospital, a } \\
\text { quality improvement initiative } \\
\text { was implemented to reduce } \\
\text { harm-causing medication } \\
\text { errors (NCC MERP D-I) in a } \\
\text { 4-year study period. The } \\
\text { initiative rendered } \\
\text { interventions to all aspects of } \\
\text { the medication use process } \\
\text { with a special focus on } \\
\text { administration errors. Error } \\
\text { data was recorded from } \\
\text { voluntary incident reporting, } \\
\text { trigger tool analysis, reversal } \\
\text { agent review, and clinical } \\
\text { pharmacist interventions }\end{array}$ & $\begin{array}{l}\text { (1) Independent } \\
\text { double-check policy for } \\
\text { high-risk medications } \\
\text { (2) Implementation of a } \\
\text { wireless nurse } \\
\text { communication system } \\
\text { (3) Smart syringes and } \\
\text { pumps with drug libraries } \\
\text { (4) Safety nurse-led audits } \\
\text { of the compliance to the } 5 \\
\text { rights } \\
\text { medication administration } \\
\text { (5) Implementation of a } \\
\text { barcoded } \\
\text { medication administration } \\
\text { (6) Pharmacy has more } \\
\text { pneumatic tubes for faster } \\
\text { delivery of compounded } \\
\text { urgent medications }\end{array}$ & Bundle & $\begin{array}{l}\text { In the } 1 \text { st quarter } 2010 \text {, } \\
\text { the number of pADEs } \\
\text { maximized to } 85 \text { within } \\
3 \text { months ( } 0.171 \text { pADE } \\
\text { per } 1000 \text { dispensed } \\
\text { doses). In the last } \\
\text { investigated quarter } \\
\text { (2nd quarter 2013), this } \\
\text { number was reduced to } \\
22 \text { pADEs within } 3 \\
\text { months (0.040 pADE } \\
\text { per } 1000 \text { dispensed } \\
\text { doses) }\end{array}$ & $0.017 \% / 0.004 \%$ & $\begin{array}{c}0.013 \% \\
\text { (significantly } \\
\text { positive results) }\end{array}$ \\
\hline
\end{tabular}


Studies addressing multiple steps in the medication use process (combined medication errors)

\begin{tabular}{|c|c|c|c|c|c|c|c|c|c|}
\hline $\begin{array}{l}\text { First author } \\
\text { (country, year) }\end{array}$ & Error type(s) & $\begin{array}{l}\text { Study design, } \\
\text { study centers }\end{array}$ & Setting & Methods & Intervention & $\begin{array}{l}\text { Single/bundle } \\
\text { intervention }\end{array}$ & Results & Error rate $\mathrm{CG} / \mathrm{IG}$ & $\begin{array}{l}\text { Absolute risk } \\
\text { reduction } \\
\text { (reported level of } \\
\text { significance) }\end{array}$ \\
\hline $\begin{array}{l}\text { Mekory et al. (Israel, } \\
\text { 2017) (73) }\end{array}$ & $\begin{array}{c}\text { Combined } \\
\text { medication } \\
\text { errors } \\
\text { (prescribing } \\
\text { errors, drug } \\
\text { administration } \\
\text { errors) }\end{array}$ & $\begin{array}{l}\text { UBA, single } \\
\text { center }\end{array}$ & $\begin{array}{c}\text { Pediatric } \\
\text { ward/emergenc } \\
\text { department }\end{array}$ & $\begin{array}{l}\text { The Joint Commission } \\
\text { cy International (JCl) } \\
\text { accreditation in } 2014 \text { was } \\
\text { sought. Therefore, a training } \\
\text { program for the } \mathrm{JCl} \text { standards } \\
\text { to preclude prescribing and } \\
\text { administration errors was } \\
\text { implemented, and a prereview } \\
\text { and post-review of } \\
\text { handwritten emergency } \\
\text { department and pediatric } \\
\text { ward medical charts were } \\
\text { performed }\end{array}$ & $\begin{array}{c}\text { Educational program } \\
\text { consisting of lectures, a } \\
\text { personal handbook, and an } \\
\text { educational software. Topics } \\
\text { discussed were prescribing } \\
\text { of an accurate order, filling it } \\
\text { by the nurse, supervising it } \\
\text { by the nurse and } \\
\text { pharmacist, and handling of } \\
\text { a medication error }\end{array}$ & Single & $\begin{array}{l}\text { Pre: during } 1 \text { month, } \\
183 \text { patients were } \\
\text { included, they got } 937 \\
\text { prescription and } 924 \\
\text { administration orders; } \\
61 \text { prescribing and } 104 \\
\text { administration errors } \\
\text { occurred; post: during } 1 \\
\text { month, } 183 \text { patients } \\
\text { were included; they got } \\
961 \text { prescription and } \\
958 \text { administration } \\
\text { orders. } 41 \text { prescribing } \\
\text { and } 114 \text { administration } \\
\text { errors occurred }\end{array}$ & $\begin{array}{c}\text { Administration } \\
\text { errors: } \\
11.3 \% / 11.9 \%\end{array}$ & $\begin{array}{l}\text { Administration } \\
\text { errors: }-0.6 \% \\
\text { (non-significant } \\
\text { negative results) }\end{array}$ \\
\hline $\begin{array}{l}\text { Migowa et al. } \\
\text { (Kenya, 2018) (74) }\end{array}$ & $\begin{array}{l}\text { Combined } \\
\text { medication } \\
\text { errors } \\
\text { (prescribing and } \\
\text { dispensing } \\
\text { errors) }\end{array}$ & $\begin{array}{l}\text { UBA, single } \\
\text { center }\end{array}$ & $\begin{array}{l}\text { EP, hospital } \\
\text { pharmacy }\end{array}$ & $\begin{array}{l}\text { Retrospective chart review of } \\
1 \text { year of prescriptions and } \\
\text { medication dispensing } \\
\text { records of physicians and } \\
\text { pharmacists in an emergency } \\
\text { department of a tertiary } \\
\text { hospital }\end{array}$ & $\begin{array}{l}\text { A voice recognition system } \\
\text { was installed at one } \\
\text { computer in the emergency } \\
\text { department. A medical } \\
\text { dictionary was developed } \\
\text { and stored in a computer } \\
\text { database; voice profiles } \\
\text { were installed; training with } \\
\text { the system was provided for } \\
\text { the users }\end{array}$ & Single & $\begin{array}{l}\text { Only duration of } \\
\text { preinterventional and } \\
\text { post-interventional } \\
\text { phase submitted: } 1 \\
\text { year; pre: } 1,196 \\
\text { prescriptions were } \\
\text { written for } 1,196 \\
\text { patients with } 889 \text { errors. } \\
\text { In the same period, } \\
1,111 \text { dispensations } \\
\text { with } 1,030 \text { errors were } \\
\text { documented; post: } 501 \\
\text { prescriptions were } \\
\text { written for } 501 \text { patients } \\
\text { with } 329 \text { errors. In the } \\
\text { same period, } 356 \\
\text { dispensations with } 332 \\
\text { errors were } \\
\text { documented; most } \\
\text { prescribing error } \\
\text { reduction was seen in } \\
\text { the dose prescription. } \\
\text { pharmacists criticized } \\
\text { that no drug database } \\
\text { existed-this may have } \\
\text { been contributed to the } \\
\text { high error rate }\end{array}$ & $\begin{array}{l}\text { Dispensing errors: } \\
\text { 92.7\%/93.3\% }\end{array}$ & $\begin{array}{c}\text { Dispensing errors: } \\
-0.5 \% \\
\text { (non-significant } \\
\text { negative results) }\end{array}$ \\
\hline
\end{tabular}




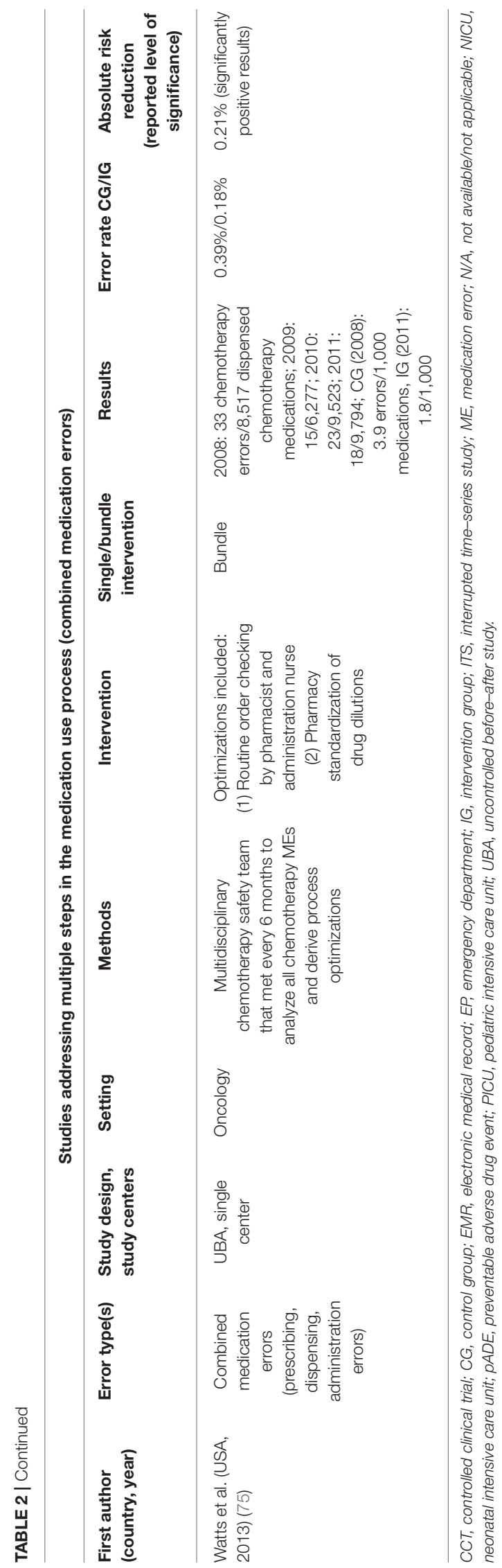

The interventions were then grouped descriptively to provide a practical overview of possible interventions at each control level.

Group comparisons were calculated using Fisher exact test (level of significance: $p<0.05$ ) or Mann-Whitney $U$-test (two-tailed, $p<0.05$ ), whatever was applicable.

Initially planned meta-analyses were deemed unfeasible because of heterogeneity in study designs, outcomes, the high number of uncontrolled before-after studies, and the high proportion of studies using a bundle of interventions, meaning the influence of one specific intervention cannot be quantified.

\section{Risk of Bias Across Studies}

All studies meeting the inclusion criteria were included, regardless of whether they reported positive, neutral, or negative results. It was assumed that there was an equal distribution for each group in case of no bias.

It has been reported that positive study results are often published sooner than non-significant or negative results, producing a time-lag bias $(79,80)$. Within this review, time to publication was assessed for each study using the time from date of data completion to the date of electronic publication $(81,82)$. Studies were grouped according to their results: into studies with significantly positive results (studies which statistically significantly reduced error rates), non-significantly results (reduced or increased error rates observed, but statistical significance not demonstrated), or mixed results. No studies demonstrating significantly negative results (increased error rates) were identified. Statistical differences were calculated using Mann-Whitney $U$-test (see above).

\section{Additional Analyses}

Definitions of medication errors were recorded and compared, following previous reports relating to heterogeneity of definitions $(6,11,16,83,84)$, which can lead to poor comparability of studies. A definition was recorded when the authors of a study clearly identified that a definition had been used (e.g., "medication error was defined as..."). Authors searched all included studies for definitions of medication error, dispensing error, drug administration error, and monitoring error.

Whether or not the error types investigated in each study were clearly defined by the study authors was also assessed.

\section{RESULTS}

\section{Study Selection}

The original search strategy was intended to identify studies investigating interventions to reduce pediatric medication errors during all stages of the MUP; the results were split posthoc to address the challenge of managing dispensing, drug administration, and monitoring errors separately to prescribing errors. Database searches identified 5,440 abstracts, which were reviewed by the first reviewer (JK) and resulted in 50 full texts that corresponded to the full inclusion criteria. A second reviewer (AE) independently assessed 547 randomly selected abstracts. This resulted in an "excellent agreement" of both reviewers (Cohen $\kappa=0.86$ ) (52). The search of the bibliographies of the included full texts as well as systematic and narrative 


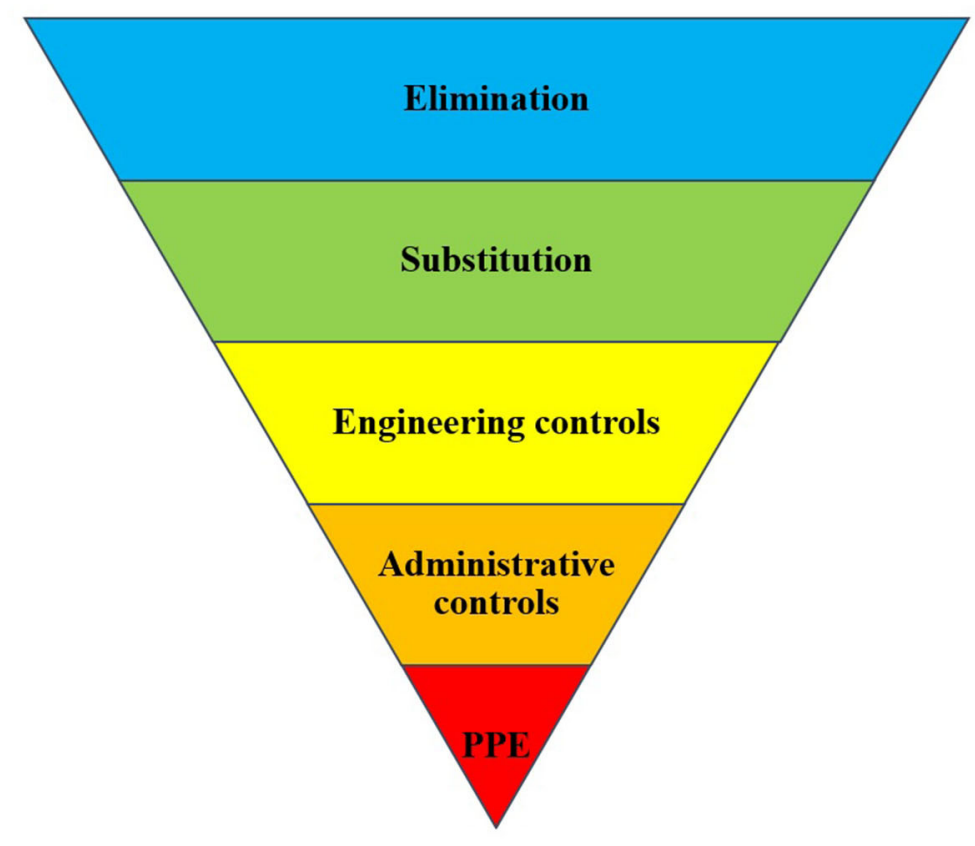

Elimination e.g. of organic solvents in tablet coating processes by producing non-coated tablets; virtually not feasible in patient care processes - even the omission of a medication would introduce the risk for a prescribing error if the medication was needed for treatment of the disease.

Substitution of a procedure with a higher risk for error by one with a lower risk for error; e.g. substitution of a manually operated infusion pump with a smart-pump containing a drug database.

Isolating the patient from the risk; e.g. Barcode Medication Administration (BCMA): the patient who would receive a medication intended for someone else is isolated from this risk for harm by a BCMA which warns the provider before administration

Tasks, which aim at behavioral change of the personnel, e.g. preparation guidelines or education

Personal protective equipment, e.g. safety glasses

Legend: $P P E=$ Personal Protective Equipment

FIGURE 1 | "Hierarchy of controls" with examples for each stage. PPE, Personal protective equipment. Adapted from: National Institute for Occupational Safety and Health (NIOSH) (76).

reviews ( $n=4,039$ abstracts) led to four additionally included publications [see PRISMA flowchart (7), Figure 2]. Of the initial search results, 20 studies met the inclusion criteria for this review, in that they investigated interventions to reduce dispensing, drug administration, and/or monitoring errors.

\section{Study Characteristics}

The 20 selected studies (56-75) originated from 11 countries and 4 continents (Table 2), with the majority being completed in North America (9/20, 45\%). One controlled clinical trial and two interrupted time-series studies were identified; the majority of studies ( $n=17 ; 85 \%$ ) were classed as uncontrolled before-after studies. All studies addressed the hospital setting, with a focus on inpatient care $(n=15,75 \%)$. Nineteen studies (95\%) were single-center studies.

\section{Risk of Bias Within Studies}

The summarized data of bias risk assessment are shown in Figures 3-5 and Supplementary Tables 2-4. All uncontrolled before-after studies had a serious or critical risk for bias due to a non-declaration of possible confounders.

\section{Results of Individual Studies}

The results of the individual studies are summarized in Table 2.

\section{Synthesis of Results} Interventions

A total of 44 different interventions were identified, which aim to reduce errors at one or more of the three included MUP stages. Eight of the included studies investigated interventions for a single point in the MUP, either dispensing or drug administration. No studies addressed monitoring errors independently of other MUP stages. The remaining 12 studies investigated interventions to reduce errors at more than one stage in the MUP and were therefore classified as combined medication error studies. The interventions in these studies, which were believed to address dispensing, drug administration, and/or monitoring, were extracted for descriptive analysis. Fourteen studies, including 34 interventions, achieved a statistically significant reduction in error rate, according to the definition of the individual study authors, 3 studies showed a statistically non-significant difference in error rates, and the remaining 3 studies reported mixed results.

\section{Interventional Approach of the Studies}

In the majority of studies $(n=13)$, the impact of a single intervention was investigated, whereas seven studies implemented a bundle of interventions. A non-statistically significant preference $(p=0.28)$ for a bundle of interventions was observed in studies targeting more than one stage of the MUP (42\% of combined medication error studies used a bundle of interventions compared with $28 \%$ of studies investigating a single step in the MUP).

\section{Single-Intervention Studies}

Only one study investigated dispensing errors at pharmacy level, independent of other stages in the MUP. This study used a single intervention in the form of the implementation of 


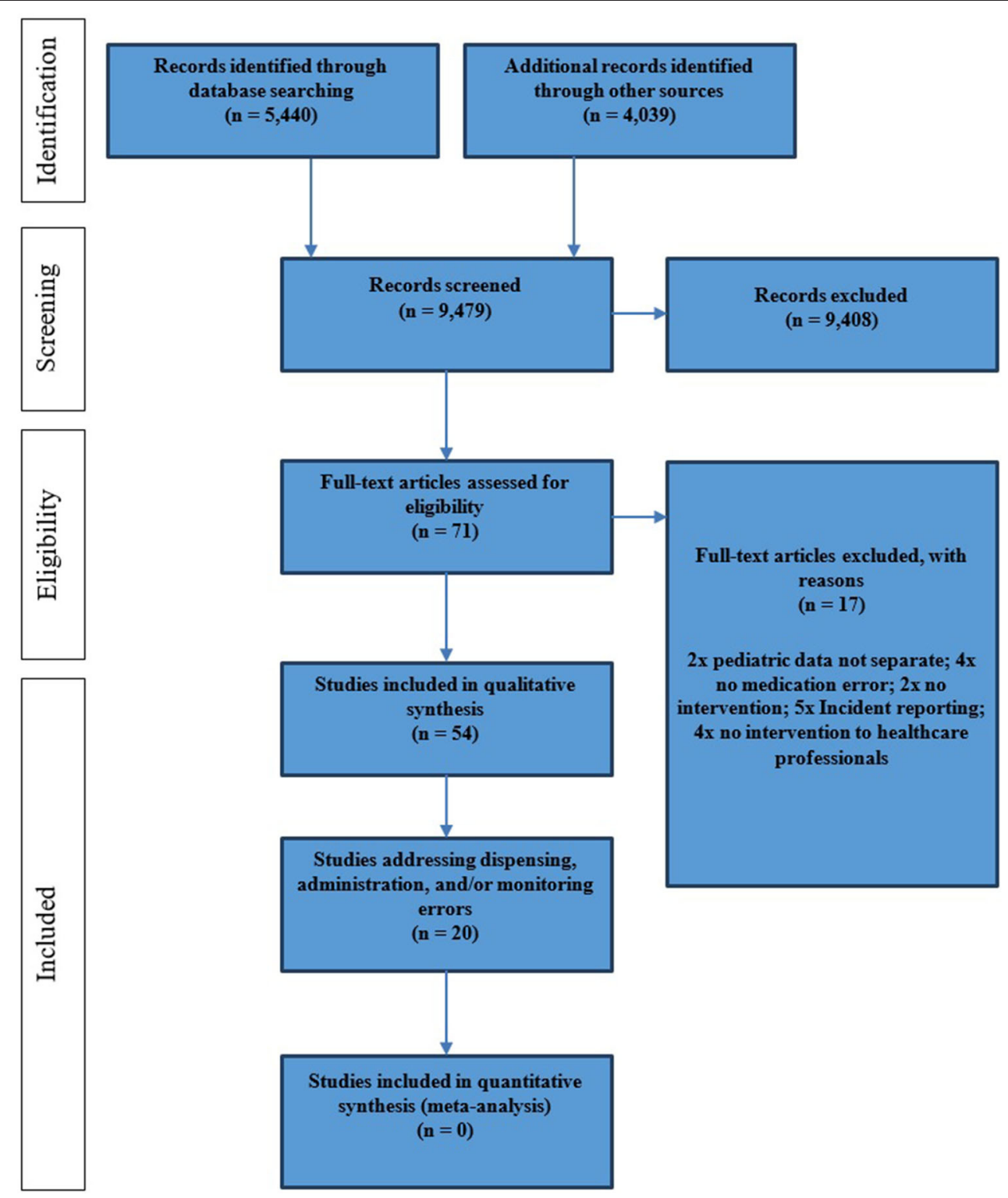

FIGURE 2 | PRISMA flowchart reporting study selection.

a new electronic workflow system, which interfaced with the electronic prescribing system already in place and demonstrated a significant reduction in error rate (59).

Five of seven studies addressing drug administration errors implemented a single intervention. Marconi et al. investigated the impact of a clinical pharmacist working in an emergency department with the aim of reducing missed or delayed medications and demonstrated an absolute risk reduction of $17.4 \%$ (60). The remaining four single-intervention studies targeting drug administration errors implemented educational approaches: Chedoe et al. combined practical and theoretical preparation and administration techniques in one educational intervention for nurses on a neonatal intensive care unit (absolute risk reduction $17.9 \%$ ) (57). Chua et al. used a pharmacist-led program involving observation of drug administration by the pharmacist followed by feedback and education to nursing and medical staff regarding observed risks or errors (absolute risk reduction $15.8 \%$ ) (58). Niemann et al. implemented a similar educational program in two different settings; a short handout was combined with a lecture and a handbook for nurses on a pediatric ward and on a pediatric intensive care unit (absolute risk reduction 7.1 and $41.0 \%$, respectively) $(61,62)$.

The single interventions implemented in the seven studies targeting multiple steps of the MUP included educational approaches (4/7), the use of computerized reminders, and the standardization of documentation $(65-68,70,73,74)$. Three of these reached significantly positive results $(65,66,70)$. 


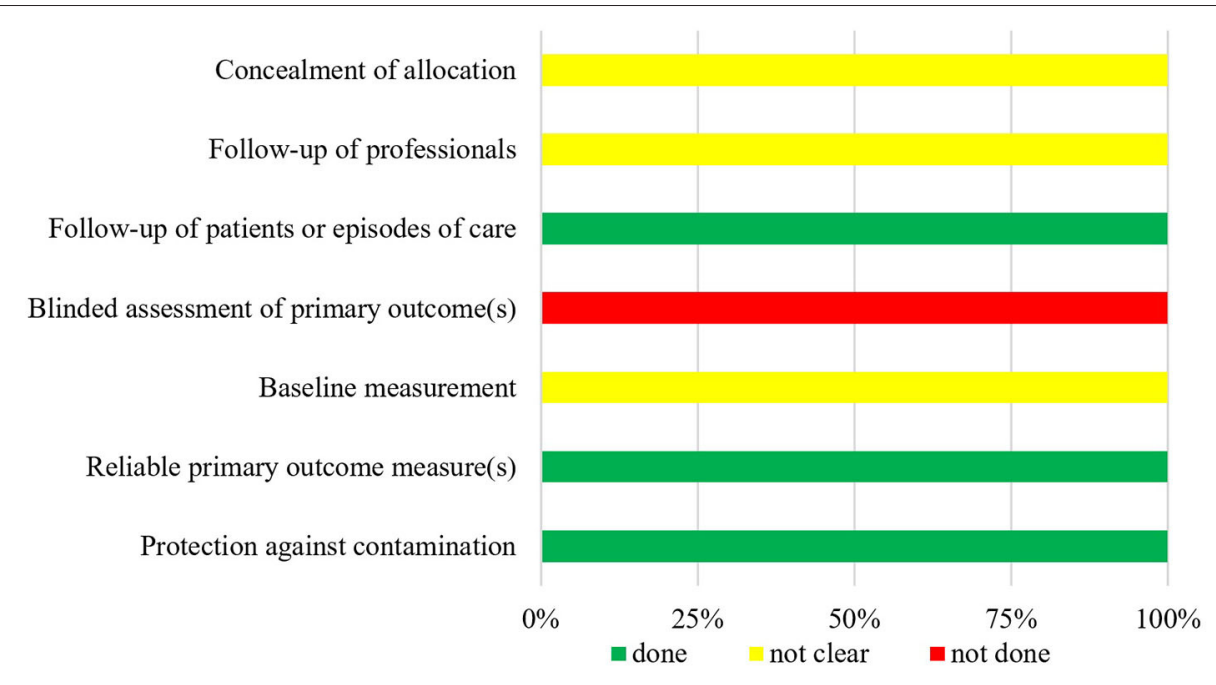

FIGURE 3 | Bias risk assessment for included CCT $(n=1)$.

The intervention is independent of other changes

Data were analyzed appropriately

Reason for the number of points pre- and post-intervention given

Shape of the intervention effect was specified

Intervention unlikely to affect data collection

Blinded assessment of primary outcome(s)

Completeness of data set

Reliable primary outcome measure(s)

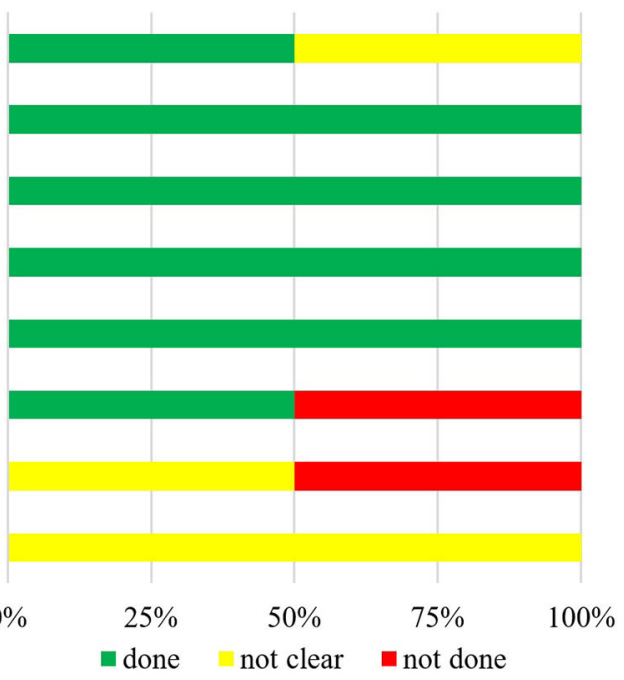

FIGURE 4 | Bias risk assessment for included ITSs $(n=2)$.

\section{Bundles of Interventions}

Seven studies introduced a bundle of interventions, namely, more than one interventional method to address one or more stages in the MUP. Bundles of interventions were wide ranging and often included an element of education $(56,63,64,69,71,72,75)$. The effect of a single intervention could not be calculated as the effects described in the studies were the result of the combination of interventions.

\section{Classification of Interventions According to the Hierarchy of Controls}

Two authors independently classified the 44 identified interventions according to the hierarchy of controls. Before discussion, agreement was $72 \%$ [weighted $\kappa=0.45$, "fair" agreement (52)]. After discussion, complete consensus was reached.
No studies were identified that eliminated the risk of error; in addition, only four interventions (9\%) were rated as having made a substitution, for example, of equipment, with the aim of reducing the risk. Seven interventions (16\%) were considered engineering controls, for example, the introduction of barcoded drug administration, whereas the vast majority of interventions were classified as "administrative controls" (33/44 interventions, $75 \%)$, such as educational programs, policies, guidelines, and warning signs (Figure 6).

Of the 44 identified interventions, 34 were implemented in the 14 studies that achieved a significant reduction in error rate. Three of the interventions involved in studies with reduced error rates were categorized as substitution controls, 7 as engineering controls, and 24 as administrative controls (Table 3 ).

Six of the seven studies that implemented higher levels of control (substitution or engineering controls) resulted in 
Bias due to confounding

Bias in selection of participants into the study

Bias in classification of interventions

Bias due to deviations from intended interventions

Bias due to missing data

Bias in measurement of outcomes

Bias in selection of the reported result

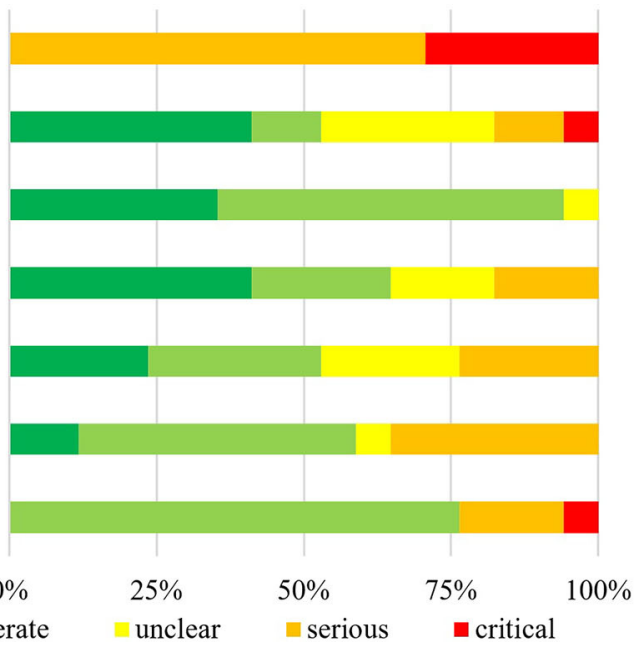

FIGURE 5 | Bias risk assessment for included UBAs $(n=17)$.

$$
\begin{array}{ccccc}
0 \% & 25 \% & 50 \% & 75 \% & 100 \% \\
\square \text { low } & \begin{array}{c}
0 \% \\
\text { moderate }
\end{array} & \text { unclear } & \square \text { serious } & \square \text { critical }
\end{array}
$$

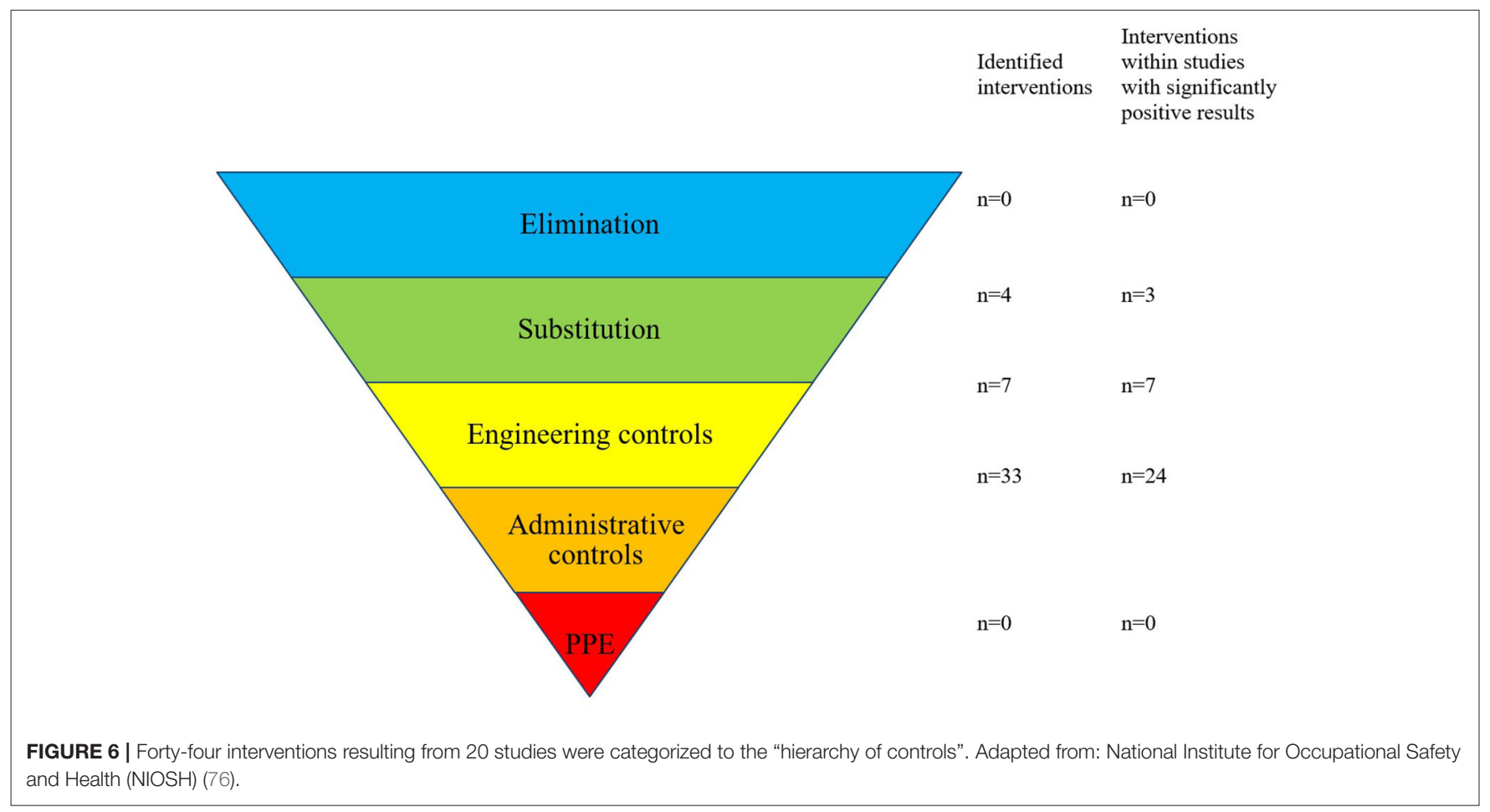

a significant reduction in error rate (86\%). In contrast, only 8 of 13 studies where solely administrative controls were implemented reported significant error reductions (62\%). Thus, studies that implemented substitution or engineering controls were 1.4 times more likely to result in reduced error rates compared to administrative controls implemented alone $(86: 62=1.4)$. This difference failed to reach statistical significance $(p=0.23)$. Studies that implemented substitution or engineering controls lasted a median of 49 months to collect control- and intervention-group data, whereas studies of administrative controls had a median duration of 4 months. The difference between these median study durations was significant (Mann-Whitney, $p<0.05$, two-tailed), demonstrating that the effects of administrative controls have been tested over shorter time periods, so the longevity of their effects is more difficult to establish.

Substitution controls included the introduction of a new pharmacy admixture unit, the introduction of smart pumps, and the use of standardized dilutions $(64,72,75)$. These substitutions all formed part of successful bundles of interventions, which led to significantly reduced error rates in combined medication error studies. The introduction of voice-recorded and printed 
TABLE 3 | Summary of interventions that were implemented in studies that significantly reduced medication errors (34 interventions of total 44 identified interventions within 14 of 20 identified studies)

\begin{tabular}{|c|c|c|}
\hline $\begin{array}{l}\text { Main risk } \\
\text { control aspect }\end{array}$ & $\begin{array}{l}\text { Number of } \\
\text { interventions in } \\
\text { studies with } \\
\text { significantly } \\
\text { positive results }\end{array}$ & Intervention type \\
\hline \multirow[t]{3}{*}{ Substitution } & $n=3$ & Standardized dilutions (75) \\
\hline & & Pharmacist production unit (64) \\
\hline & & Smart pumps (72) \\
\hline \multirow{5}{*}{$\begin{array}{l}\text { Engineering } \\
\text { controls }\end{array}$} & $n=7$ & Electronic workflow/CPOE (59) \\
\hline & & $\begin{array}{l}\text { Enhanced medication delivery } \\
\text { equipment (72) }\end{array}$ \\
\hline & & $\begin{array}{l}\text { Hands-free communication } \\
\text { equipment }(2 \times)(69,72)\end{array}$ \\
\hline & & $\begin{array}{l}\text { Barcoded medication } \\
\text { administration }(2 \times)(69,72)\end{array}$ \\
\hline & & Computerized alert (66) \\
\hline \multirow[t]{5}{*}{$\begin{array}{l}\text { Administrative } \\
\text { controls }\end{array}$} & $n=24$ & $\begin{array}{l}\text { Education and/or practical training } \\
(8 \times)(56-58,61,64,69,72)^{\star}\end{array}$ \\
\hline & & $\begin{array}{l}\text { Guidelines or protocols }(6 \times) \\
(56,63,65,69,72,75)\end{array}$ \\
\hline & & $\begin{array}{l}\text { Rearrangement of staff or } \\
\text { equipment }(3 \times)(63,64)^{\dagger}\end{array}$ \\
\hline & & $\begin{array}{l}\text { Expert consultation }(4 \times) \\
(60,64,70)^{\ddagger}\end{array}$ \\
\hline & & Warning signs $(3 \times)(63,69)^{\S}$ \\
\hline
\end{tabular}

*Keiffer et al. implemented two different educational interventions (medication error huddles and "5 rights education").

${ }^{\dagger}$ Ozkan et al. implemented two different rearrangement interventions (decrease of patientto-nurse ratio and modified delivery time of medications).

${ }^{\ddagger}$ Abuelsoud et al. implemented two different expert consultation interventions (implementation of a clinical pharmacist within the medical team and a drug information service).

$\S$ Ozkan et al. implemented two different warning sign interventions (written alert on the door of the preparation room and signaling arm bands for medication-preparing nurses).

prescriptions did not lead to a reduced rate of dispensing errors (74).

Seven engineering controls were identified within four different studies $(59,66,69,72)$; five of these controls were implemented as parts of bundles of interventions, alongside administrative controls. They were demonstrated in all cases to be effective through a significant reduction in errors. Interventions included the introduction of a workflow management system, additional supplies to facilitate the use of pneumatic tubes, hands-free communication systems, and barcoded drug administration. Alerts in the patient electronic medical record were also successful on this occasion, although carefully implemented to reduce the risk of alert fatigue, which was acknowledged by the authors.

The overwhelming majority of interventions identified were classified as administrative controls. They could be subdivided into "education and training" $(n=12)$, "guidelines, protocols and procedures" $(n=8)$, "rearrangement of staff/material" $(n=6)$, “expert consultations" $(n=4)$, and "warning signs” $(n=3)$.

\section{Risk of Bias Across Studies}

Based on the high percentage of significantly positive results (70\%), a publication bias could not be ruled out.

Sixteen studies (80\%) reported a date when data collection was completed, allowing the calculation of time to publication. Studies reporting significantly positive results were published after a median of 26 months (interquartile range $=17.5-37$ months), whereas mixed results were published after a median of 39 months (interquartile range $=33.25-44.5$ months). When compared to the distribution of significant positive results, a statistically significant difference could not be confirmed.

\section{Additional Analyses}

In 14 of 20 full texts, 16 definitions were identified within 44 opportunities for definition (36\%, see Supplementary Table 5). Seven definitions of a medication error (35\% of all included full texts), one definition of a dispensing error $(33 \%$ for a total of 3 full texts), seven definitions of a drug administration error (39\%, total: 18 studies), and one definition of a monitoring error (33\% for a total of 3 full texts) were identified. These definitions were heterogeneous in content and often failed to consider the patient; e.g., the definition for "medication error" contained only in one case the phrase "preventability"; the definitions for "drug administration error" did not contain any patient-centered aspect and had instead a technical focus.

\section{DISCUSSION}

\section{Summary of Evidence}

We identified 44 individual interventions designed to reduce dispensing, drug administration, and monitoring errors and classified them according to a hierarchical approach to risk control. This is, to our knowledge, the first review to use the hierarchy of controls model to classify interventions for improving pediatric medication safety. This model, adapted from the health and safety industry, suggests that interventions should first be sought at the highest level of control (elimination of risk) before other options that are lower on the hierarchy and therefore potentially less effective are considered $(78,85)$. Although none of the interventions identified in this review were classified by the authors at the highest level (elimination), our results do support the theory that interventions at higher levels (substitution and engineering) should be prioritized, through the observation that studies implementing higher-level controls were 1.4 times more likely to achieve a significant reduction in error rates than those using administrative controls only. This is in line with the findings of Card et al., who reported a ratio of 1.6 (78). Despite this, we have observed that administrative controls are more frequently implemented, indicating a potential opportunity to rethink our approach to risk reduction and quality improvement, with a focus first on the opportunities to substitute risks or use engineering controls $(77,78)$, in favor of the perhaps easier-to-implement administrative controls.

There are criticisms of the use of a hierarchical control model in this setting. The lack of consideration of the potential human factors involved in medication errors has been cited as a key challenge (86), and Liberati et al. judged that "this model adds 
little value to the development of effective risk controls in clinical settings and lacks validity and usefulness" (87). The possibility of truly eliminating risks from the MUP can also be questioned, because omission of one or more medications would in itself be a risk to the patient (88). Practically seen, we may therefore expect interventions, which substitute a risk-prone process or step with a lower-risk option to be the strongest type of intervention available to us in the field of medication safety. We suggest that the hierarchy of controls model could be a useful tool to prompt those designing interventions to first consider the higher levels of control, before opting to implement administrative controls. We must, however, also acknowledge that the complexity of the MUP requires the use of a wider range of quality improvement tools and methodologies in order to design effective interventions, which also take local factors into consideration (85).

The wide range of interventions identified in this review at the higher levels of control supports this consideration. These interventions can be broadly considered as fitting into one of two groups. The first group includes interventions that interact with electronic medical records or electronic prescribing systems, which were already in place in the included studies $(59,66,69$, 72). On this basis, we would suggest that electronic prescribing and patient records provide a good foundation on which other interventions can be developed. The second group of higherlevel interventions includes those that interact with the working environment, often with the aim of reducing interruptions and/or redistributing workload among the multidisciplinary team, for example, medication production being carried out by the pharmacy team in a specialized unit, rather than by the nursing team on the ward $(64,69,72,75)$. These improvements may lead to reduced error-producing conditions and in turn to improved pediatric medication safety (89).

The majority of the identified interventions were classified as administrative controls. Interestingly, we noted that these measures were often implemented alongside higher-level controls, as parts of bundles of interventions. In these studies, it is not possible to separate the influence of individual interventions within the bundle, and we must therefore acknowledge the potential importance of administrative controls including education, guidelines, and protocols when implemented alongside higher-level interventions.

In the review at hand, similar limitations of the currently published literature were identified as have been shown in previous systematic reviews in the field $(6,11,34,44)$. The included publications mainly originated from North America or Europe; the studies were performed primarily in inpatient settings, and the identified definitions of central terms were of heterogeneous nature. These factors should be considered, particularly when planning future research projects in this area.

\section{STRENGTH AND LIMITATIONS}

We used the hierarchy of controls model for the first time to classify interventions to reduce pediatric dispensing, drug administration, and monitoring errors and have demonstrated that this model may be an appropriate tool for use in this setting.

Our methodology is potentially limited as the initial data extraction was performed by one researcher, with corrections and/or additions being provided by a second independent reviewer. As most studies were uncontrolled before-after studies and therefore present a high risk of bias, the results should be interpreted with caution. The inclusion criteria comprised studies published in English only, leading to a potential foreign language bias, although evidence for this is questionable (90). In addition, this review includes experimental studies for an 8-year time span only and must therefore be interpreted alongside the results of previous, and future subsequent, reviews on the same topic.

\section{CONCLUSION}

When designing interventions to reduce pediatric dispensing, drug administration, and monitoring errors, the hierarchy of controls model should be considered, with a focus placed on the introduction of higher-level controls, which may be more likely to reduce errors than the administrative controls often seen in practice. A wide range of approaches to addressing the risks of dispensing and administering medications for pediatric patients in inpatient professional healthcare settings has been identified, and it is important to consider local conditions when planning interventions.

\section{DATA AVAILABILITY STATEMENT}

The original contributions presented in the study are included in the article/Supplementary Material, further inquiries can be directed to the corresponding author/s.

\section{AUTHOR CONTRIBUTIONS}

JK was responsible for search, data extraction/analysis, and drafted the first manuscript. NY assisted in the data analysis. $\mathrm{UK}, \mathrm{TO}$, and $\mathrm{DB}$ contributed to interpretation of data. AE developed the concept, supervised, and assisted with data extraction/analysis. JK, NY, UK, TO, DB, and AE revisited the manuscript critically for important intellectual content. They approved the final manuscript as submitted and take full responsibility for the manuscript. All authors are accountable for all aspects of the work and for ensuring that questions related to the accuracy or integrity of any part of the work are appropriately investigated and resolved.

\section{SUPPLEMENTARY MATERIAL}

The Supplementary Material for this article can be found online at: https://www.frontiersin.org/articles/10.3389/fped. 2021.633064/full\#supplementary-material 


\section{REFERENCES}

1. World Health Organization. Medication Safety in High-risk Situations. (2019). Available online at: https://apps.who.int/iris/bitstream/handle/10665/325131/ WHO-UHC-SDS-2019.10-eng.pdf?ua=1 (accessed October 15, 2020).

2. World Health Organization. Promoting Safety of Medicines for Children. (2007). Available online at: https://www.who.int/medicines/publications/ essentialmedicines/Promotion_safe_med_childrens.pdf (accessed November 02, 2020).

3. Kaushal R, Bates DW, Landrigan C, McKenna KJ, Clapp MD, Federico F, et al. Medication errors and adverse drug events in pediatric inpatients. JAMA. (2001) 285:2114-20. doi: 10.1001/jama.285.16.2114

4. National Coordinating Council for Medication Error Reporting and Prevention. About Medication Errors. (2016). Available online at: http://www. nccmerp.org/about-medication-errors (accessed May 23, 2016).

5. Aitken M, Gorokhovich L. Advancing the Responsible Use of Medicines: Applying Levers for Change. Danbury, CT, SSRN 2222541 (2012). doi: 10.2139/ssrn.2222541

6. Rinke ML, Bundy DG, Velasquez CA, Rao S, Zerhouni Y, Lobner K, et al. Interventions to reduce pediatric medication errors: a systematic review. Pediatrics. (2014) 134:338-60. doi: 10.1542/peds.2013-3531

7. Liberati A, Altman DG, Tetzlaff J, Mulrow C, Gotzsche PC, Ioannidis JP, et al. The PRISMA statement for reporting systematic reviews and meta-analyses of studies that evaluate health care interventions: explanation and elaboration. PLoS Med. (2009) 6:e1000100. doi: 10.1371/journal.pmed.1000100

8. Franklin BD, O'Grady K. Dispensing errors in community pharmacy: frequency, clinical significance and potential impact of authentication at the point of dispensing. Int J Pharm Pract. (2007) 15:273-81. doi: 10.1211/ijpp.15.4.0004

9. Ghaleb MA, Barber N, Franklin BD, Wong IC. The incidence and nature of prescribing and medication administration errors in paediatric inpatients. Archiv Dis Child. (2010) 95:113-8. doi: 10.1136/adc.2009.158485

10. Alldred D, Standage C, Zermansky A. Development and validation of criteria to identify medication-monitoring errors in care home residents. Int $J$ Pharm Pract. (2008) 16:317-23. doi: 10.1211/ijpp.16.5.0007

11. Maaskant JM, Vermeulen H, Apampa B, Fernando B, Ghaleb MA, Neubert A, et al. Interventions for reducing medication errors in children in hospital. Cochrane Database System Rev. (2015) 3:Cd006208. doi: 10.1002/14651858.CD006208.pub3

12. Manias E, Kinney S, Cranswick N, Williams A, Borrott N. Interventions to reduce medication errors in pediatric intensive care. Ann Pharmacother. (2014) 48:1313-31. doi: 10.1177/1060028014543795

13. Parand A, Garfield S, Vincent C, Franklin BD. Carers' medication administration errors in the domiciliary setting: a systematic review. PLoS ONE. (2016) 11:e0167204. doi: 10.1371/journal.pone.0167204

14. Krzyzaniak N, Bajorek B. Medication safety in neonatal care: a review of medication errors among neonates. Ther Adv Drug Saf. (2016) 7:10219. doi: $10.1177 / 2042098616642231$

15. Berdot S, Roudot M, Schramm C, Katsahian S, Durieux P, Sabatier B. Interventions to reduce nurses' medication administration errors in inpatient settings: a systematic review and meta-analysis. Int J Nurs Stud. (2016) 53:342-50. doi: 10.1016/j.jinurstu.2015.08.012

16. Bannan DF, Tully MP. Bundle interventions used to reduce prescribing and administration errors in hospitalized children: a systematic review. J Clin Pharm Ther. (2016) 41:246-55. doi: 10.1111/jcpt.12398

17. Wimmer S, Neubert A, Rascher W. The safety of drug therapy in children. Dtsch Arztebl. (2015) 112:781-7. doi: 10.3238/arztebl.2015.0781

18. Santesteban E, Arenas S, Campino A. Medication errors in neonatal care: a systematic review of types of errors and effectiveness of preventive strategies. J Neonatal Nurs. (2015) 21:200-8. doi: 10.1016/j.jnn.2015.04.002

19. Chan HK, Hassali MA, Lim CJ, Saleem F, Tan WL. Using pictograms to assist caregivers in liquid medication administration: a systematic review. J Clin Pharm Ther. (2015) 40:266-72. doi: 10.1111/jcpt.12272

20. Bower R, Jackson C, Manning JC. Interruptions and medication administration in critical care. Nurs Crit Care. (2015) 20:18395. doi: $10.1111 /$ nicc. 12185

21. Alomari A, Wilson V, Davidson PM, Lewis J. Families, nurses and organisations contributing factors to medication administration error in paediatrics: a literature review. Int Pract Develop J. (2015) 5:1-14. doi: 10.19043/ipdj.51.007

22. Nuckols TK, Smith-Spangler C, Morton SC, Asch SM, Patel VM, Anderson LJ, et al. The effectiveness of computerized order entry at reducing preventable adverse drug events and medication errors in hospital settings: a systematic review and meta-analysis. Syst Rev. (2014) 3:56. doi: 10.1186/2046-4053-3-56

23. Neuspiel DR, Taylor MM. Reducing the risk of harm from medication errors in children. Health Services Insights. (2013) 6:47-59. doi: 10.4137/HSI.S10454

24. Huynh C, Wong IC, Tomlin S, Terry D, Sinclair A, Wilson K, et al. Medication discrepancies at transitions in pediatrics: a review of the literature. Paediatr Drugs. (2013) 15:203-15. doi: 10.1007/s40272-013-0030-8

25. Garfield S, Reynolds M, Dermont L, Franklin BD. Measuring the severity of prescribing errors: a systematic review. Drug Saf. (2013) 36:1151-7. doi: 10.1007/s40264-013-0092-0

26. Mehndiratta S. Strategies to reduce medication errors in pediatric ambulatory settings. J Postgr Med. (2012) 58:47-53. doi: 10.4103/0022-3859.93252

27. Meguerdichian MJ, Clapper TC. The Broselow tape as an effective medication dosing instrument: a review of the literature. J Pediatr Nurs. (2012) 27:41620. doi: 10.1016/j.pedn.2012.04.009

28. Kaufmann J, Laschat M, Wappler F. Medication errors in pediatric emergencies: a systematic analysis. Deutsches Arzteblatt Int. (2012) 109:60916. doi: 10.3238/arztebl.2012.0609

29. Alsulami Z, Conroy S, Choonara I. Double checking the administration of medicines: what is the evidence? A systematic review. Archiv Dis Child. (2012) 97:833-7. doi: 10.1136/archdischild-2011-301093

30. Wilson S, Bremner A, Hauck Y, Finn J. The effect of nurse staffing on clinical outcomes of children in hospital: a systematic review. Int J Evidence Based Healthcare. (2011) 9:97-121. doi: 10.1111/j.1744-1609.2011.00209.x

31. Davis T. Paediatric prescribing errors. Archiv Dis Child. (2011) 96:48991. doi: $10.1136 /$ adc. 2010.200295

32. Alghamdi AA, Keers RN, Sutherland A, Ashcroft DM. Prevalence and nature of medication errors and preventable adverse drug events in paediatric and neonatal intensive care settings: a systematic review. Drug Saf. (2019) 42:142336. doi: 10.1007/s40264-019-00856-9

33. Alsaidan J, Portlock J, Aljadhey HS, Shebl NA, Franklin BD. Systematic review of the safety of medication use in inpatient, outpatient and primary care settings in the Gulf Cooperation Council countries. Saudi Pharmac J. (2018) 26:977-1011. doi: 10.1016/j.jsps.2018.05.008

34. Drovandi A, Robertson K, Tucker M, Robinson N, Perks S, Kairuz T. A systematic review of clinical pharmacist interventions in paediatric hospital patients. Eur J Pediatr. (2018) 177:1139-48. doi: 10.1007/s00431-018-3187-x

35. Feinstein MM, Pannunzio AE, Castro P. Frequency of medication error in pediatric anesthesia: a systematic review and meta-analytic estimate. Paediatr Anaesth. (2018) 28:1071-7. doi: 10.1111/pan.13521

36. Gates PJ, Meyerson SA, Baysari MT, Lehmann CU, Westbrook JI. Preventable adverse drug events among inpatients: a systematic review. Pediatrics. (2018) 142:e20180805. doi: 10.1542/peds.2018-0805

37. Gates PJ, Meyerson SA, Baysari MT, Westbrook JI. The prevalence of dose errors among paediatric patients in hospital wards with and without health information technology: a systematic review and meta-analysis. Drug Saf. (2019) 42:13-25. doi: 10.1007/s40264-018-0715-6

38. Gates PJ, Baysari MT, Gazarian M, Raban MZ, Meyerson S, Westbrook JI. Prevalence of medication errors among paediatric inpatients: systematic review and meta-analysis. Drug Saf. (2019) 42:1329-42. doi: 10.1007/s40264-019-00850-1

39. Kahn S, Abramson EL. What is new in paediatric medication safety? Arch Dis Child. (2019) 104:596-9. doi: 10.1136/archdischild-2018-315175

40. Kaufmann J, Wolf AR, Becke K, Laschat M, Wappler F, Engelhardt T. Drug safety in paediatric anaesthesia. Br J Anaesth. (2017) 118:6709. doi: $10.1093 / \mathrm{bja} / \mathrm{aex} 072$

41. Koyama AK, Maddox CS, Li L, Bucknall T, Westbrook JI. Effectiveness of double checking to reduce medication administration errors: a systematic review. BMJ Qual Saf. (2020) 29:595-603. doi: 10.1136/bmjqs-2019009552

42. Leung JS, Johnson DW, Sperou AJ, Crotts J, Saude E, Hartling L, et al. A systematic review of adverse drug events associated with administration of common asthma medications in children. PLoS ONE. (2017) 12:e0182738. doi: 10.1371/journal.pone.0182738 
43. Melton KR, Ni Y, Tubbs-Cooley HL, Walsh KE. Using health information technology to improve safety in neonatal care: a systematic review of the literature. Clin Perinatol. (2017) 44:583-616. doi: 10.1016/j.clp.2017.04.003

44. Nguyen MR, Mosel C, Grzeskowiak LE. Interventions to reduce medication errors in neonatal care: a systematic review. Ther Adv Drug Saf. (2018) 9:123-55. doi: 10.1177/2042098617748868

45. Patel NS, Patel TK, Patel PB, Naik VN, Tripathi CB. Hospitalizations due to preventable adverse reactions-a systematic review. Eur J Clin Pharmacol. (2017) 73:385-98. doi: 10.1007/s00228-016-2170-6

46. Prgomet M, Li L, Niazkhani Z, Georgiou A, Westbrook JI. Impact of commercial computerized provider order entry (CPOE) and clinical decision support systems (CDSSs) on medication errors, length of stay, and mortality in intensive care units: a systematic review and meta-analysis. J Am Med Inform Assoc. (2017) 24:413-22. doi: 10.1093/jamia/ocw145

47. Roumeliotis N, Sniderman J, Adams-Webber T, Addo N, Anand V, Rochon $\mathrm{P}$, et al. Effect of electronic prescribing strategies on medication error and harm in hospital: a systematic review and meta-analysis. J Gen Int Med. (2019) 34:2210-23. doi: 10.1007/s11606-019-05236-8

48. Rush KL, Howlett L, Munro A, Burton L. Videoconference compared to telephone in healthcare delivery: a systematic review. Int J Med Inform. (2018) 118:44-53. doi: 10.1016/j.ijmedinf.2018.07.007

49. Sutherland A, Phipps DL, Tomlin S, Ashcroft DM. Mapping the prevalence and nature of drug related problems among hospitalised children in the United Kingdom: a systematic review. BMC Pediatr. (2019) 19:486. doi: 10.1186/s12887-019-1875-y

50. Wells M, Goldstein LN, Bentley A, Basnett S, Monteith I. The accuracy of the Broselow tape as a weight estimation tool and a drug-dosing guide - a systematic review and meta-analysis. Resuscitation. (2017) 121:933. doi: 10.1016/j.resuscitation.2017.09.026

51. York JB, Cardoso MZ, Azuma DS, Beam KS, Binney GG, Jr., et al. Computerized physician order entry in the neonatal intensive care unit: a narrative review. Appl Clin Inform. (2019) 10:487-94. doi: 10.1055/s-0039-1692475

52. Orwin RG. Evaluating coding decisions. In: H. Cooper, LV Hedges, editors. The Handbook of Research Synthesis. New York, NY: Russell Sage Foundation (1994). p. 139-62.

53. Cochrane Effective Practice Organisation of Care Review Group. Data Collection Checklist. (2002). Available online at: https://epoc.cochrane. org/sites/epoc.cochrane.org/files/public/uploads/datacollectionchecklist.pdf (accessed November 24, 2020).

54. Cochrane Effective Practice and Organisation of Care Review Group. Data Abstraction Form. (2012). Available online at: https://epoc.cochrane.org/ sites/epoc.cochrane.org/files/uploads/Data\%20Abstraction\%20Form\%20\%20EPOC\%20-\%202012-Feb-23.doc (accessed February 07, 2017).

55. Sterne JA, Hernan MA, Reeves BC, Savovic J, Berkman ND, Viswanathan M, et al. ROBINS-I: a tool for assessing risk of bias in non-randomised studies of interventions. BMJ. (2016) 355:i4919. doi: 10.1136/bmj.i4919

56. Campino A, Santesteban E, Pascual P, Sordo B, Arranz C, Unceta M, et al. Strategies implementation to reduce medicine preparation error rate in neonatal intensive care units. Eur J Pediatr. (2016) 175:75565. doi: 10.1007/s00431-015-2679-1

57. Chedoe I, Molendijk H, Hospes W, Van den Heuvel ER, Taxis K. The effect of a multifaceted educational intervention on medication preparation and administration errors in neonatal intensive care. Arch Dis Child Fetal Neonatal Ed. (2012) 97:F449-55. doi: 10.1136/fetalneonatal-2011-300989

58. Chua SS, Choo SM, Sulaiman CZ, Omar A, Thong MK. Effects of sharing information on drug administration errors in pediatric wards: a pre-post intervention study. Ther Clin Risk Manag. (2017) 13:34553. doi: 10.2147/TCRM.S128504

59. Davis SJ, Hurtado J, Nguyen R, Huynh T, Lindon I, Hudnall C, et al. Innovations in medication preparation safety and wastage reduction: use of a workflow management system in a pediatric hospital. Hospital Pharm. (2017) 52:54-9. doi: 10.1310/hpj5201-54

60. Marconi GP, Claudius I. Impact of an emergency department pharmacy on medication omission and delay. Pediatr Emergency Care. (2012) 28:303. doi: 10.1097/PEC.0b013e31823f232d

61. Niemann D, Bertsche A, Meyrath D, Koepf ED, Traiser C, Seebald K, et al. A prospective three-step intervention study to prevent medication errors in drug handling in paediatric care. J Clin Nurs. (2015) 24:10114. doi: 10.1111 /jocn. 12592

62. Niemann D, Bertsche A, Meyrath D, Oelsner S, Ewen AL, Pickardt B, et al. Drug handling in a paediatric intensive care unit-can errors be prevented by a three-step intervention? Klinische Padiatrie. (2014) 226:627. doi: $10.1055 / \mathrm{s}-0033-1364030$

63. Ozkan S, Kocaman G, Ozturk C. Effect of strategies for preventing medication administration errors in pediatric inpatients. Turk Pediatri Arsivi. (2013) 48:299-302. doi: 10.4274/tpa.540

64. Abuelsoud N. Pharmacy quality improvement project to enhance the medication management process in pediatric patients. Irish J Med Sci. (2019) 188:591-600. doi: 10.1007/s11845-018-1860-8

65. Benkelfat R, Gouin S, Larose G, Bailey B. Medication errors in the management of anaphylaxis in a pediatric emergency department. $J$ Emergency Med. (2013) 45:419-25. doi: 10.1016/j.jemermed.2012.11.069

66. Ernst KD. Electronic alerts improve immunization rates in two-month-old premature infants hospitalized in the neonatal intensive care unit. Appl Clin Inform. (2017) 8:206-13. doi: 10.4338/ACI-2016-09-RA-0156

67. Fawaz MG, Sabri NA, Albaghdady AA, Malek FAA. Detection and prevention of medication errors in the operating rooms of a pediatric surgery department in Egypt. Int J Pharm Sci Rev Res. (2017) 42:100-6.

68. Foster ME, Lighter DE, Godambe AV, Edgerson B, Bradley R, Godambe S. Effect of a resident physician educational program on pediatric emergency department pharmacy interventions and medication errors. J Pediatr Pharmacol Ther. (2013) 18:53-62. doi: 10.5863/1551-6776-18.1.53

69. Keiffer S, Marcum G, Harrison S, Teske DW, Simsic JM. Reduction of medication errors in a pediatric cardiothoracic intensive care unit. J Nurs Care Qual. (2015) 30:212-9. doi: 10.1097/NCQ.0000000000000098

70. Maaskant JM, Tio MA, van Hest RM, Vermeulen H, Geukers VGM. Medication audit and feedback by a clinical pharmacist decrease medication errors at the PICU: an interrupted time series analysis. Health Sci Rep. (2018) 1:e23. doi: $10.1002 / \mathrm{hsr} 2.23$

71. Martin LD, Grigg EB, Verma S, Latham GJ, Rampersad SE, Martin LD. Outcomes of a failure mode and effects analysis for medication errors in pediatric anesthesia. Paediatr Anaesth. (2017) 27:571-80. doi: 10.1111/pan.13136

72. McClead RE Jr., Catt C, Davis JT, Morvay S, Merandi J, Lewe D, et al. An internal quality improvement collaborative significantly reduces hospital-wide medication error related adverse drug events. J Pediatr. (2014) 165:1222-9.e1. doi: 10.1016/j.jpeds.2014. 08.063

73. Mekory TM, Bahat H, Bar-Oz B, Tal O, Berkovitch M, Kozer E. The proportion of errors in medical prescriptions and their executions among hospitalized children before and during accreditation. Int J Qual Health Care. (2017) 29:366-70. doi: 10.1093/intqhc/mzx031

74. Migowa AN, Macharia WM, Samia P, Tole J, Keter AK. Effect of a voice recognition system on pediatric outpatient medication errors at a tertiary healthcare facility in Kenya. Ther Adv Drug Saf. (2018) 9:499508. doi: $10.1177 / 2042098618781520$

75. Watts RG, Parsons K. Chemotherapy medication errors in a pediatric cancer treatment center: prospective characterization of error types and frequency and development of a quality improvement initiative to lower the error rate. Pediatr Blood Cancer. (2013) 60:1320-4. doi: 10.1002/pbc.24514

76. The National Institute for Occupational Safety and Health. Hierarchy of Controls. (2015). Available online at: https://www.cdc.gov/niosh/topics/ hierarchy/default.html (accessed October 13, 2020).

77. Card AJ, Ward JR, Clarkson PJ. Generating Options for Active Risk Control (GO-ARC): introducing a novel technique. J Healthcare Qual. (2014) 36:3241. doi: 10.1111/jhq. 12017

78. Card AJ, Ward J, Clarkson PJ. Successful risk assessment may not always lead to successful risk control: a systematic literature review of risk control after root cause analysis. J Healthc Risk Manag. (2012) 31:612. doi: 10.1002/jhrm. 20090

79. Stern JM, Simes RJ. Publication bias: evidence of delayed publication in a cohort study of clinical research projects. BMJ. (1997) 315:6405. doi: 10.1136/bmj.315.7109.640

80. Hopewell S, Loudon K, Clarke MJ, Oxman AD, Dickersin K Publication bias in clinical trials due to statistical significance 
or direction of trial results. Cochrane Database Syst Rev. (2009) Mr000006. doi: 10.1002/14651858.MR000006.pub3

81. Ioannidis JP. Effect of the statistical significance of results on the time to completion and publication of randomized efficacy trials. JAMA. (1998) 279:281-6. doi: 10.1001/jama.279.4.281

82. Reyes MM, Panza KE, Martin A, Bloch MH. Time-lag bias in trials of pediatric antidepressants: a systematic review and meta-analysis. J Am Acad Child Adolesc Psychiatry. (2011) 50:63-72. doi: 10.1016/j.jaac.2010.10.008

83. Chedoe I, Molendijk HA, Dittrich ST, Jansman FG, Harting JW, Brouwers JR, et al. Incidence and nature of medication errors in neonatal intensive care with strategies to improve safety: a review of the current literature. Drug Saf. (2007) 30:503-13. doi: 10.2165/00002018-200730060-00004

84. Sanghera N, Chan PY, Khaki ZF, Planner C, Lee KK, Cranswick NE, et al. Interventions of hospital pharmacists in improving drug therapy in children: a systematic literature review. Drug Saf. (2006) 29:103147. doi: 10.2165/00002018-200629110-00003

85. Manuele FA. Risk assessment \& hierarchies of control: their growing importance to the SH\&E profession. Profess Saf. (2005) 50:33-9.

86. Reason J. Human error: models and management. BMJ. (2000) 320:76870. doi: $10.1136 /$ bmj. 320.7237 .768

87. Liberati EG, Peerally MF, Dixon-Woods M. Learning from high risk industries may not be straightforward: a qualitative study of the hierarchy of risk controls approach in healthcare. Int J Qual Health Care. (2018) 30:3943. doi: $10.1093 /$ intqhe/mzx163
88. National Coordinating Council for Medication Error Reporting and Prevention. NCC MERP Index for Categorizing Medication Errors. (2001). Available online at: http://www.nccmerp.org/sites/default/files/ indexColor2001-06-12.pdf (accessed July 20, 2016).

89. Sanghera IS, Franklin BD, Dhillon S. The attitudes and beliefs of healthcare professionals on the causes and reporting of medication errors in a UK intensive care unit. Anaesthesia. (2007) 62:53-61. doi: 10.1111/j.1365-2044.2006.04858.x

90. Morrison A, Polisena J, Husereau D, Moulton K, Clark M, Fiander M, et al. The effect of English-language restriction on systematic review-based metaanalyses: a systematic review of empirical studies. Int J Technol Assess Health Care. (2012) 28:138-44. doi: 10.1017/S0266462312000086

Conflict of Interest: The authors declare that the research was conducted in the absence of any commercial or financial relationships that could be construed as a potential conflict of interest.

Copyright (๑) 2021 Koeck, Young, Kontny, Orlikowsky, Bassler and Eisert. This is an open-access article distributed under the terms of the Creative Commons Attribution License (CC BY). The use, distribution or reproduction in other forums is permitted, provided the original author(s) and the copyright owner $(s)$ are credited and that the original publication in this journal is cited, in accordance with accepted academic practice. No use, distribution or reproduction is permitted which does not comply with these terms. 